\title{
A Stochastic Approach for the Analysis of \\ Dynamic Fault Trees with Spare Gates under Probabilistic Common Cause Failures
}

Peican Zhu, Jie Han, Leibo Liu and Fabrizio Lombardi

Abstract - A redundant system usually consists of primary and standby modules as critical components for fault tolerance. The so-called spare gate is extensively used to model the dynamic behavior of such a system in the analysis of dynamic fault trees (DFTs). Several methodologies have been proposed to evaluate the reliability of DFTs containing spare gates by computing the failure probability. However, either a complex analysis or a significant simulation time is usually required by such an approach. Moreover, it is difficult to compute the top event's failure probability for basic events that are not exponentially distributed. Additionally, probabilistic common cause failures (PCCFs) have been widely reported, usually occurring in a dependent manner. Failure to account for the effect of PCCFs overestimates the reliability of a DFT. In this paper, stochastic computational models are proposed for an efficient analysis of spare gates and PCCFs in a DFT. Using these models, a DFT with spare gates under PCCFs can be efficiently evaluated. In the proposed stochastic approach, a signal probability is encoded as a non-Bernoulli sequence of random permutations of fixed numbers of $1 \mathrm{~s}$ and $0 \mathrm{~s}$. The basic event's failure probability is not limited to an exponential distribution, thus this approach is applicable to a DFT analysis in a general case. Several case studies are evaluated to show the accuracy and efficiency of the 
proposed approach, compared to both an analytical approach and Monte Carlo (MC) simulation.

Index Terms - Dynamic fault tree, warm spare gate (WSP), cold spare gate (CSP), hot spare gate (HSP), reliability analysis, stochastic computation, non-Bernoulli sequence, stochastic logic, probabilistic common cause failure (PCCF).

\section{ACRONYM}

$\begin{array}{ll}\text { FTA } & \text { fault tree analysis } \\ \text { DFT } & \text { dynamic fault tree } \\ \text { FDEP } & \text { functional dependency gate } \\ \text { PAND } & \text { priority AND gate } \\ \text { SEQ } & \text { sequence enforcing gate } \\ \text { WSP } & \text { warm spare gate } \\ \text { CSP } & \text { cold spare gate } \\ \text { BSP } & \text { hot spare gate } \\ p d f & \text { probability density function decision diagrams } \\ & \end{array}$


MC Monte Carlo

FPGA field programmable gate array

CCF common cause failure

PCCF probabilistic common cause failure

\section{NOTATION}

$\begin{array}{ll}\rightarrow & \text { an inclusive precedence in failure order } \\ t & \text { mission time } \\ A, B, C & \text { basic events } \\ \lambda & \text { failure rate } \\ p\left(t_{i}\right) & \text { binary sequence at time } t_{i} \text { generated for } p\left(t_{i}\right) \\ S\left(t_{i}\right) & \text { sequence length in bits for the stochastic approach }\end{array}$

\section{INTRODUCTION}

$\mathrm{F}^{\text {AULT tree analysis (FTA) was first proposed in the } 1960 \text { s for evaluating the reliability of a }}$ flight system [1]. Over the last few decades, this technique has been widely applied to the analysis of various systems, including chemical plants, nuclear reactors, airplane controllers and computers [2]. The so-called dynamic fault tree (DFT) has been developed to mimic the dynamic behavior of a system; this has been accomplished by incorporating several additional dynamic gates, such as the priority AND gate (PAND), the sequence enforcing gate (SEQ), the standby or 
spare gate (SPARE) and the functional dependency gate (FDEP) $[3,4,5]$.

Various approaches have been proposed to analyze DFTs, including Markov models [6, 7], Bayesian [8], an Inclusion/Exclusion approach [9], a numerical integration approach [10], binary decision diagrams (BDDs) [11], sequential binary decision diagrams (SBDDs) [12, 13] and Monte Carlo (MC) simulation $[14,15]$.

In a redundant system, some modules are online or operational, while one or more modules function as standby. They are therefore referred to as primary and standby modules, respectively. Standby modules are critical for tolerating hardware failures or software errors; this is achieved by removing the faulty primary module from the operation and replacing it with a spare unit [16]. In a DFT, the primary and standby modules are considered as input events to a spare gate [13]. If the primary and standby components are not treated as basic events, or the input events include several standby components, it becomes cumbersome to obtain the failure probability through the use of existing approaches. Recently, stochastic computational approaches using random binary bit streams have been proposed for the reliability analysis of logic circuits $[17,18]$ and DFT analysis [19]. It has been shown that the use of non-Bernoulli sequences of random permutations of fixed umbers of $1 \mathrm{~s}$ and $0 \mathrm{~s}$ as initial inputs leads to an efficient and accurate evaluation by a stochastic approach [18]. A stochastic model for PAND gates has been proposed for use in an efficient DFT analysis [20]. As shown in [20], signal correlations are inherently preserved in stochastic sequences; hence, repeated events are readily accounted. Furthermore, the general case of nonexponential distributions is modeled efficiently by a stochastic approach [20]. For systems with perfect fault coverage, the FDEP can be treated as an OR gate [21, 22]; the SEQ gate can be regarded as a special case of a cold spare gate (CSP) [23]. Furthermore, a hot spare gate (HSP) is logically equivalent to an AND gate [13]. The priority relationship is considered in the stochastic 
PAND model of [20]; however, modeling of spare gates (and in particular, the warm spare gate (WSP) and CSP), has not been considered. Hence, this paper focuses on the WSP and CSP gates. A stochastic model is first proposed for spare gates in the analysis of DFTs; both exponential and non-exponential (e.g. Weibull) distributions are then analyzed using this stochastic model.

In practice, the basic events of a system are often subject to common cause failures (CCFs) including earthquakes, sudden changes in the environment, design errors and incorrect operations [24]. CCFs are sometimes closely related; for instance, floods are likely to be caused by hurricanes. These CCFs are referred to as dependent CCFs. Furthermore, the occurrence of a CCF is usually not deterministic, but probabilistic, thus referred to as a probabilistic CCF (PCCF) [25]. The probability of occurrence differs by components or conditions. The consideration of PCCFs in a DFT analysis is of great significance as the system's reliability is likely to be overestimated without incorporating PCCFs. However, it presents a great challenge to consider PCCFs in a DFT analysis using existing methods, such as an integration-based approach. In this paper, a stochastic model is proposed for modeling the effect of dependent PCCFs. A general DFT with independent/dependent PCCFs can be efficiently evaluated by the proposed stochastic approach. The accuracy of a stochastic analysis increases with the length of the non-Bernoulli sequences in stochastic computation. In summary, this paper makes the following novel contributions:

1. Stochastic computational models for WSP and CSP, as well as for CCFs and PCCFs.

2. An efficient analysis of DFTs using stochastic models for different static and dynamic gates by incorporating CCFs and PCCFs; both exponential and non-exponential failure distributions are considered for the failures of basic events.

The remainder of this paper is organized as follows. Section II presents some hypotheses considered in this work. Section III presents a review of spare gates. Section IV first introduces 
the fundamentals of the stochastic approach and a non-Bernoulli sequence generation algorithm; then, stochastic models are proposed for spare gates, majority voters and CCFs/PCCFs. Several benchmarks are simulated and analyzed in Section V. Finally, Section VI concludes the paper.

\section{Assumptions}

The following assumptions are made in this paper:

- The quantization level of a basic event is represented as a binary variable $x, x \in\{0,1\}$, with 0 indicating no fault;

- All basic events are fault-free at the beginning of the mission time;

- The basic events are assumed to be non-repairable [26];

- The failure probability of a component in a selected time interval $\left[t_{i}, t_{i}+\Delta t\right]$ is considered constant at the value at the beginning of the time interval, i.e., the failure probability is given by $p=F\left(t_{i}\right)$ for any time in the considered time interval. For simplicity, the time interval $\left[t_{i}, t_{i}+\Delta t\right]$ is referred to as time $i$ in this paper.

\section{REVIEW OF SPARE GATES}

A standby system modeled by a spare gate usually consists of two types of modules: the primary (or online) modules and the standby modules. Standby modules are used to replace the faulty modules to keep the system functional or operational. Hence, the spare gate fires (i.e., fails) if both of the modules fail. Spare gates are divided into three categories, depending on the switching relationship of the primary and standby modules: the hot spare gate (HSP) $[13,26]$, the cold spare gate (CSP) [13, 26], and the warm spare gate (WSP) [26, 27, 28, 29]. In an HSP system, a standby module is always powered and ready to replace a faulty primary module when a fault occurs. HSPs are typically used in systems, in which a minimal reconfiguration time is required, 
e.g. a chemical process control system. In a CSP, however, the standby modules are usually not powered until it is necessary to replace a faulty module [30]. Hence, it is usually used in power consumption critical systems, such as a satellite system [16]. As a tradeoff between CSP and HSP, the standby modules in a WSP are powered initially, but with a lower failure rate. The failure rate of the standby module in a WSP changes when it is switched to replace a faulty module [27]. For WSP, usually less power is consumed in the standby state compared to HSP, and less initialization and recovery time are required compared to CSP [28, 29].

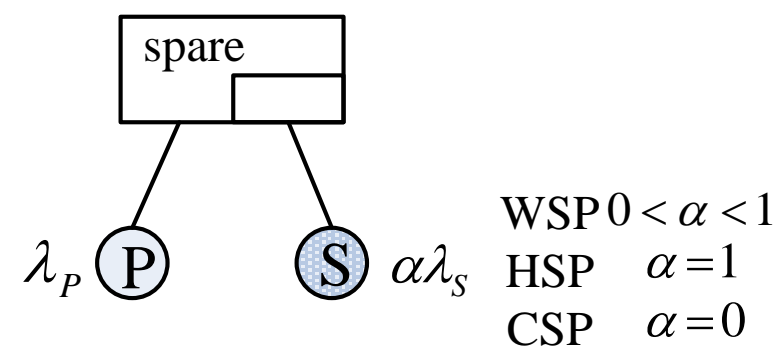

Fig. 1. A spare gate [26]. It is classified into different categories (WSP, HSP and CSP) by the factor $\alpha$, according to the failure behavior of the standby module.

Fig. 1 shows different types of spare gate. A spare gate models the sequential failure events of the primary online module, $P$, (with a failure rate of $\lambda_{P}$ ) and the standby module, $S$. The failure rate is assumed to be $\alpha \cdot \lambda_{s}$ prior to the switching of the standby module to replace a faulty component. The standby module in operation is subject to a failure rate $\lambda_{S}$ after switching. Hence, the spare gate can be classified by a different value of the factor $\alpha$ : if $\alpha=1$, the gate is an HSP gate; if $0<\alpha<1$, it becomes a WSP gate; if $\alpha=0$, it is a CSP gate. A generic failure rate switching diagram for a spare gate is shown in Fig. 2.

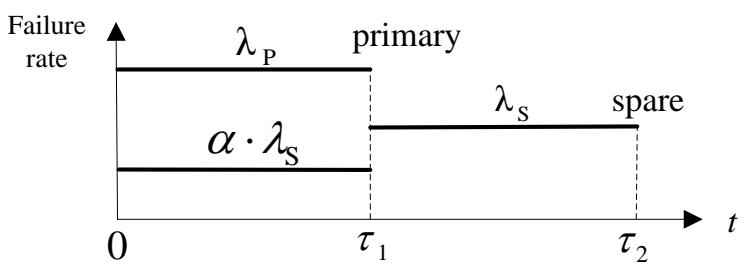


Fig. 2. A generic switching diagram for the failure in a spare gate $[12,26]$.

HSP and CSP gates can be regarded as special cases of the WSP gate; the only difference lies in the value of the failure rate before and after the switching point. A spare gate can be converted into a combinational fault tree with two sequential components serving as inputs of an OR gate, as shown in Fig. $3[12,13]$.

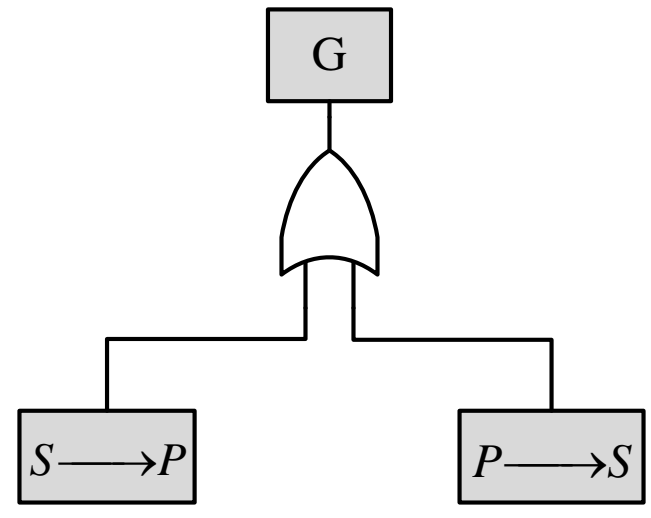

(a)

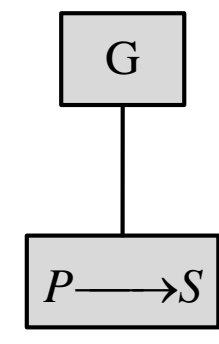

(b)

Fig. 3. The spare gate decomposition: (a) A combinational model for the spare gate, and (b) A simplified model for CSP. " $\rightarrow$ " indicates an inclusive precedence in a failure order.

In Fig. 3, the sequential event $S \rightarrow P$ indicates that both modules fail and the standby module fails before the primary module does; while the sequential event $P \rightarrow S$ means that both modules fail and the primary module fails before the standby module. The two sequential events cannot occur at the same time, thus they are mutually exclusive. Furthermore, it is impossible for the standby module of a CSP gate to fail because the failure rate of the standby module before switching is 0 . Hence, the combinational model for a CSP gate can be simplified as shown in Fig. 3(b); this indicates that the output failure probability of a CSP gate is the same as the failure probability of the sequential event $P \rightarrow S$. The output failure probability of the spare gate in Fig. 3(a) is given by [12]:

$$
U_{\text {sys }}=p(P \rightarrow S)+p(S \rightarrow P)
$$


while the probabilities of the two sequential failure events in (1) are given by:

$$
p(P \rightarrow S)=\int_{0}^{t} \int_{0}^{t-\tau_{2}} f_{P}\left(\tau_{2}\right) f_{S, \lambda}\left(\tau_{1}\right)\left(1-\int_{0}^{\tau_{2}} f_{S, \alpha \lambda}\left(\tau_{1}\right) d \tau_{1}\right) d \tau_{2} d \tau_{1},
$$

and

$$
p(S \rightarrow P)=\int_{0}^{t} \int_{\tau_{1}}^{t} f_{P}\left(\tau_{2}\right) f_{S, \alpha \lambda}\left(\tau_{1}\right) d \tau_{2} d \tau_{1}
$$

where $f_{A}(t), f_{S, \alpha \lambda}(t)$ and $f_{S, \lambda}(t)$ are the failure probability density functions ( $\left.p d f s\right)$ for the primary module, the standby module before replacing the faulty primary module and after replacing the faulty primary module. For a k-out-of-n WSP system consisting of identical WSPs, a closed expression can be derived; however, it is only applicable to systems with identical input events [31].

\section{Proposed Stochastic Models}

\section{A. Stochastic Computation}

Stochastic computation was initially proposed in the 1960s for reliable circuit design [32]. Probabilities are encoded into random binary bit streams by setting a proportional number of bits to a specific value, i.e., 1 or 0 . By using stochastic logic, Boolean logic operations are transformed into probabilistic computations in the real domain. Stochastic computation has the advantages of hardware simplicity and fault tolerance. However, inevitable random fluctuations occur in the computation of probabilities. Conventionally, Bernoulli sequences are utilized as random binary bit streams in stochastic computation. In a Bernoulli sequence, every bit is independently generated as 1 or 0 , according to a specified probability. For a probability of 0.5 , this process is similar to a coin-flipping experiment, i.e. a head or tail is observed for approximately half of the trials. Due to its probabilistic nature, the number of $1 \mathrm{~s}$ or $0 \mathrm{~s}$ in a Bernoulli sequence is not deterministic, so stochastic fluctuations exist in the computed result. [18] has shown that the use of non-Bernoulli 
sequences of fixed numbers of $1 \mathrm{~s}$ and $0 \mathrm{~s}$ for the initial input probabilities significantly reduces the effect of stochastic fluctuations compared to Bernoulli sequences. In this type of non-Bernoulli sequences [18], the numbers of $1 \mathrm{~s}$ and $0 \mathrm{~s}$ are computed from a specified probability, and then they are randomly permuted to encode the probability. This is a more efficient process compared to the generation of Bernoulli sequences, because less pseudo-random numbers need to be generated. The non-Bernoulli sequences contain deterministic numbers of $1 \mathrm{~s}$ and $0 \mathrm{~s}$, so there is no variation in the initial sequences. Therefore, the use of non-Bernoulli sequences as initial inputs results in less variation in the stochastic computing process of a network, thus it produces more accurate results than the case when Bernoulli sequences are used as initial inputs.

Examples of computation and encoding using non-Bernoulli sequences are shown in Fig. 4(a-d) for a sequence length of 10 bits; a longer sequence length is usually required in a practical application, as shown in Fig. 4(e). For the 2-to-1 multiplexer of Fig. 4(e), the output takes the value of one of the two inputs when the control bit is 0 or 1 . When stochastic sequences are used as input and control signals, this multiplexer selects one of the inputs as output according to the distributions (and thus the probabilities) of $0 \mathrm{~s}$ and $1 \mathrm{~s}$ in the control sequence. In a stochastic implementation, the multiplexer takes one of the inputs as output according to the probabilities encoded in the distributions of the control bits.

Furthermore, the repeated input events (as typically encountered in an FTA) are readily dealt with in a stochastic approach because a stochastic computing technique efficiently handles the problem of signal re-convergence [20], as shown in Fig 4(c). The stochastic logic gates used in this work are shown as follows: 


$$
\begin{aligned}
& \frac{0000111001}{P_{1}=0.4} \frac{1111000110}{P=0.6} \\
& P=1-P_{1}=0.6 \\
& P_{1}=0.9 \\
& \begin{array}{l}
\hline 1111101111 \\
\hline P=0.8
\end{array} \\
& P_{2}=0.9 \\
& P=P_{1} \cdot P_{2}=0.8 \\
& P_{1}=1111111011=\frac{1111111011}{P=0.9} \\
& P=P_{1}=P_{2}=0.9
\end{aligned}
$$

(c)

$$
\begin{aligned}
& P_{1}=0.5 \\
& \hline \begin{array}{l}
1110000101 \\
P_{2}=0.8 \\
P=1-\left(1-P_{1}\right) \cdot\left(1-P_{2}\right)=0.9
\end{array}
\end{aligned}
$$

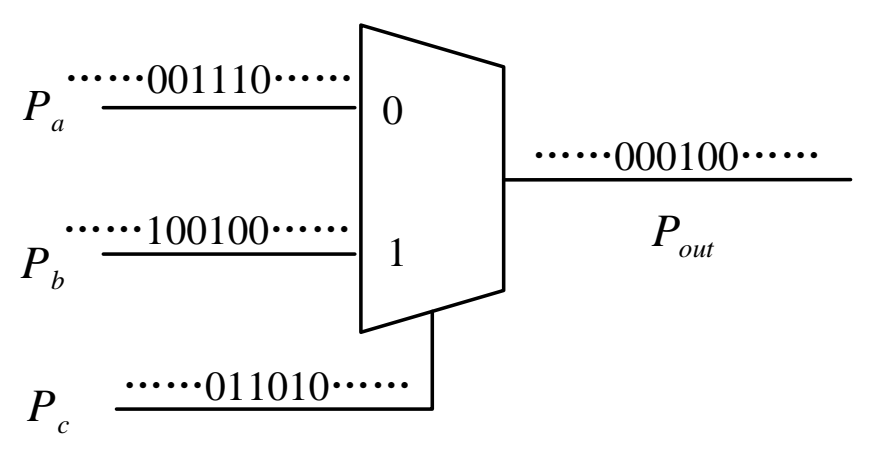

(e)

Fig. 4. Stochastic logic: (a) An inverter with a random binary bit sequence as input, (b) An AND gate with independent inputs, (c) an AND gate with totally dependent inputs, (d) An OR gate with independent inputs, (e) A 2-to-1 multiplexer.

\section{B. Stochastic models for spare gates}

The discretization of a continuous probability distribution and the generation of the nonBernoulli sequences are introduced next, followed by a stochastic model for the spare gate.

\section{1) Discretization}

Given the probability density function $(p d f)$ of a failure, $f(t)$, the cumulative density function $(c d f), F(t)$, is calculated as $F(t)=\int_{0}^{t} f(t) d t$. Similar to [20], the mission time $t$ is divided into $M$ equal time intervals; $M$ is determined by a tradeoff between accuracy and efficiency. The discretization provides a relatively accurate estimate of the failure probability of a 
basic event with a reasonable $M$.

\section{2) Generation of non-Bernoulli sequences}

Let the failure probabilities for two adjacent time intervals, time $i-1$ and time $i$, be denoted by $p_{i-1}$ and $p_{i}$ respectively. Since the non-Bernoulli sequences use random permutations of fixed numbers of 1 s and 0 s, for a sequence length of $L$ bits the number of 1 s in the sequences for the two failure probabilities are determined by $N\left(p_{i-1}\right)=L \cdot p_{i-1}$ and $N\left(p_{i}\right)=L \cdot p_{i}$ respectively. Let the non-Bernoulli sequence for the probability at time $i-1$ be represented by $S\left(p_{i-1}\right)$, then the sequence $S\left(p_{i}\right)$ for the probability at time $i$ can be obtained by randomly assigning a number of $1 \mathrm{~s}$ to replace the $0 \mathrm{~s}$ in $S\left(p_{i-1}\right)$; this number is given by $\Delta N=N\left(p_{i}\right)-$ $N\left(p_{i-1}\right)=L \cdot\left(p_{i}-p_{i-1}\right)$. The relationship between the two non-Bernoulli sequences for two adjacent time intervals is then given by:

$$
S\left(p_{i}\right) A N D S\left(p_{i-1}\right)=S\left(p_{i-1}\right)
$$

(4) is due to the assumption of non-reparability, such that the $1 \mathrm{~s}$ in $S\left(p_{i-1}\right)$ still remain as $1 \mathrm{~s}$ in $S\left(p_{i}\right)$; thus, the mutual set in both sequences is given by $S\left(p_{i-1}\right)$.

\section{3) Stochastic model of the WSP/CSP gate}

The failure probability of the spare gate with any inputs is given by (1); however, it is more complex to derive the exact failure probability for non-exponentially distributed basic events. Those are generally more realistic to model a basic event's failure behavior in a mechanical system. The derivation process becomes even more cumbersome when the primary and standby components are combinations of several other events. Moreover, the components in a realistic DFT system may also suffer from common cause failures that occur either deterministically or probabilistically. This makes the distribution of the failure behavior even more complicated. Hence, a stochastic model is proposed in this paper for efficiently analyzing spare gates. 
Let $S_{i-1}^{P}$ and $S_{i}^{P}$ denote the non-Bernoulli sequences generated for the failure probabilities of the primary module at two adjacent time intervals $i-1$ and $i$, i.e., $F_{i-1}^{P}$ and $F_{i}^{P}$, where $F^{P}$ is the $c d f$ for the failure of the primary module. As a primary module is non-repairable, the relationship of (4) must be met. For the $j$ th bit in the non-Bernoulli sequence, the state of the primary module is given by $S_{i-1, j}^{P}$ and $S_{i, j}^{P}$ for the two consecutive time intervals: a state of 0 or 1 indicates that no fault occurs or a fault occurs. The state combination of the primary module at time $i-1$ and time $i$ for the $j$ th trial - a trial is carried out by a bit or a combination of bits in the stochastic sequences - is represented by $S_{i-1, j}^{P} S_{i, j}^{P}$, where $S_{i-1, j}^{P} S_{i, j}^{P} \in\{00,01,11\}$, due to the non-reparability assumption. For the WSP/CSP gate, the failure rate of the standby module varies before and after switching to replace the primary module (for CSP, the failure probability is 0 before switching). Hence, it is necessary to record the failure time of the primary module to determine the failure probability of the standby module. If $S_{k-1, j}^{P}=0$ and $S_{k, j}^{P}=1$, it indicates that the primary module fails at time $k$ for the $j$ th trial; hence, for WSP and CSP, the operational time of the standby module should be determined from the failure time of the primary module, i.e., $t_{s}=i-k$, where $i$ is the present mission time and $k$ is the failure time of the primary module. Similarly, the operational time of the standby module can be determined for any other trial.

Let $S_{i-1}^{S}$ and $S_{i}^{S}$ be the stochastic sequences generated for the failure probabilities of the standby module at two adjacent time intervals $i-1$ and $i$. Then we discuss the following three different cases when $S_{i-1, j}^{P} S_{i, j}^{P}=00, S_{i-1, j}^{P} S_{i, j}^{P}=01$ and $S_{i-1, j}^{P} S_{i, j}^{P}=11$ :

- For $S_{i-1, j}^{P} S_{i, j}^{P}=00$, the primary module does not fail at time $i$. For a WSP, if $S_{i-1, j}^{S}=1$, then $S_{i, j}^{S}=1$. If $S_{i-1, j}^{S}=0$, the current state of the standby module for the $j$ th trial is determined by the failure probability, i.e., the $c d f F_{i}^{S, \alpha \lambda}$ obtained from the failure rate of the 
standby module before it is switched to replace the primary module.

- For $S_{i-1, j}^{P} S_{i, j}^{P}=01$, the primary module fails at time $i$ and the standby module is expected to replace the faulty primary module at the failure time. For a WSP, hence, if $S_{i-1, j}^{S}=0, S_{i, j}^{S}$ is determined by the failure $c d f$ of the standby module before switching to replace the faulty module, $F_{i}^{S, \alpha \lambda}$, because the primary module still functions at time $i-1$.

In both of these two cases, $S_{i, j}^{S}$ remains 0 for a CSP as the standby module is not activated prior to the failure of the primary module and is assumed to be fault free before it is switched to replace the faulty primary module.

- For $S_{i-1, j}^{P} S_{i, j}^{P}=11$, the primary module has failed by time $i-1$. For the standby module, if $S_{i-1, j}^{S}=1$, then $S_{i, j}^{S}=1$. Otherwise, $S_{i-1, j}^{S}$ is determined for a WSP gate by the failure $c d f$, i.e. $F_{t_{s}}^{S, \lambda}$, obtained from the failure rate of the standby module after switching to replace the faulty primary module; while for a CSP, it is also determined by $F_{t_{s}}^{S, \lambda}$, where $t_{s}$ is the operational time of the standby module.

These processes are shown in the flowchart of Fig. 5(a), which can be implemented by the stochastic architecture in Fig. 5(b). These stochastic architectures model the sequential behavior of the WSP/CSP gates. 


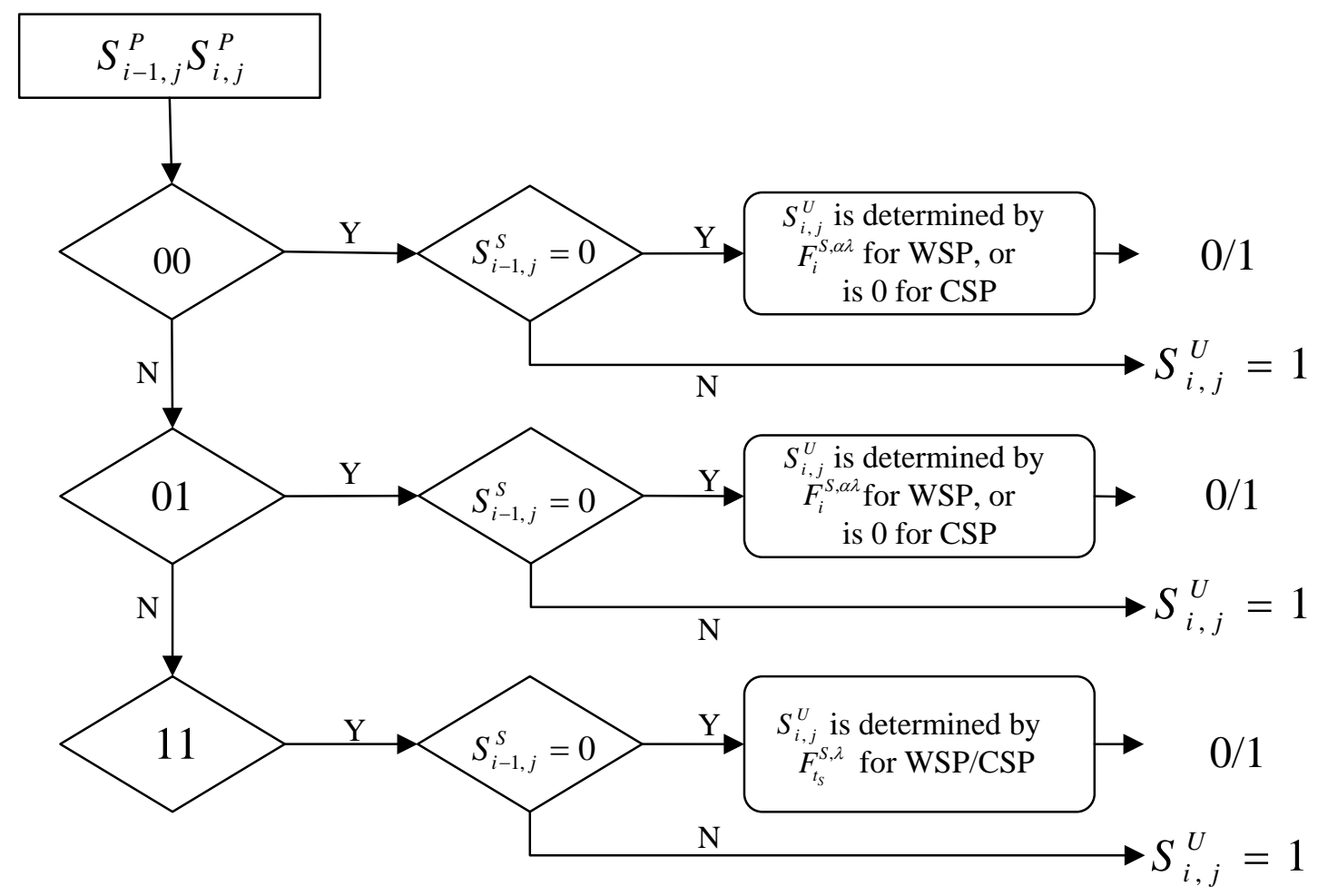

(a)

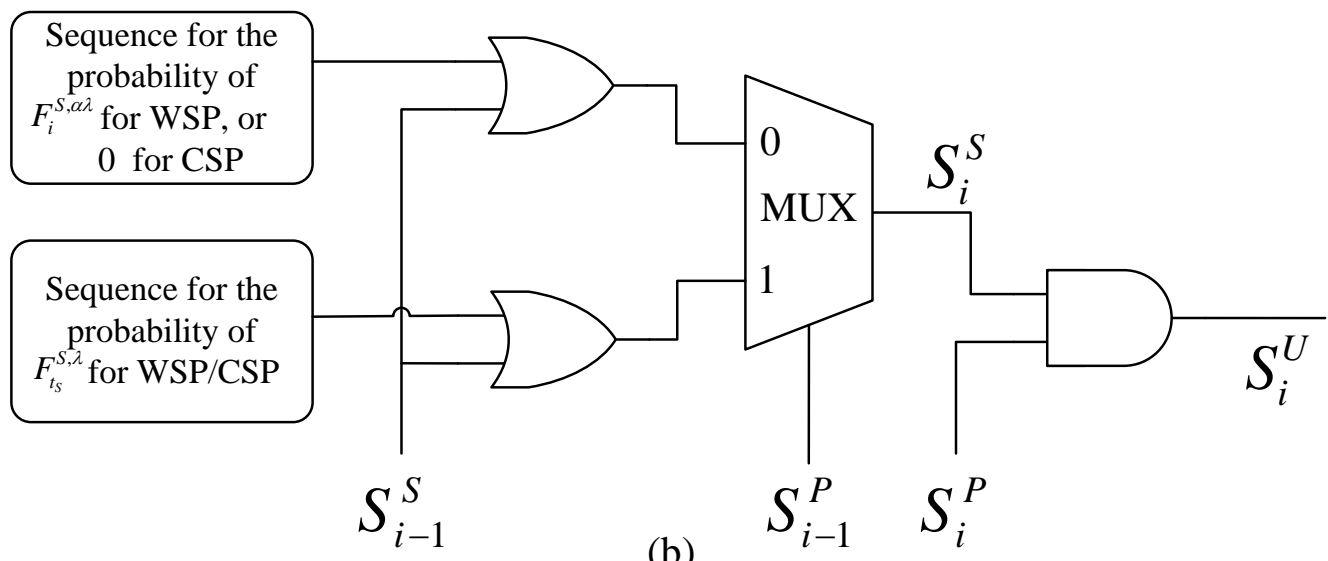

Fig. 5 (a) Flowchart for generating the stochastic sequences of the standby module, and (b) A general stochastic logic model for the spare gates (WSP and CSP). $S_{i}^{U}$ denotes the output sequence for the spare gate.

The proposed stochastic model is applied to evaluate the WSP in Fig. 1; the results are compared with those obtained by an accurate approach [12], as shown in Table 1. In Table 1, the failures of basic events are exponentially distributed with $\lambda_{P}=0.001, \alpha=0.6$, and $\lambda_{S}=0.0025$. $\boldsymbol{F}_{A}$ and $\boldsymbol{F}_{S}$ are the failure probability vectors for the accurate and stochastic analysis respectively. 
$\Delta \boldsymbol{F}_{S-A}$ denotes the discrepancies of the two failure probability vectors, i.e., $\Delta \boldsymbol{F}_{S-A}=\boldsymbol{F}_{S}-\boldsymbol{F}_{A}$. The differences between the simulation results are measured by several norms. For a vector $\boldsymbol{x}$, the norms are defined as $\|x\|_{1}=\sum_{i=1}^{n}\left|x_{i}\right|,\|x\|_{2}=\sqrt{\sum_{i=1}^{n}\left|x_{i}\right|^{2}}$ and $\|x\|_{\infty}=\max _{1 \leq i \leq n}\left|x_{i}\right|$, where $n$ is the number of elements in the vector $\boldsymbol{x}$.

Table 1 Evaluation of the stochastic WSP gate model for a mission time of 1000 hours compared with an accurate approach [12]. The average simulation time for the stochastic approach is also provided.

\begin{tabular}{c|c|c|c}
\hline \hline Sequence length $L$ (bits) & $L=1 \mathrm{k}$ & $L=10 \mathrm{k}$ & $L=100 \mathrm{k}$ \\
\hline$\left\|\Delta \boldsymbol{F}_{S-A}\right\|_{1}$ & 6.0512 & 2.1548 & 0.8354 \\
\hline$\left\|\Delta \boldsymbol{F}_{S-A}\right\|_{2}$ & 0.2424 & 0.0763 & 0.0163 \\
\hline$\left\|\Delta \boldsymbol{F}_{S-A}\right\|_{\infty}$ & 0.0189 & 0.0047 & 0.0025 \\
\hline Average run time (s) & 0.2385 & 1.0893 & 10.477 \\
\hline \hline
\end{tabular}

For the WSP gate with the same initial parameters of Table 1, the top event's failure probability is obtained for different mission times by the accurate and stochastic approaches (for a sequence length of 10k). For the WSP gate, the exact failure probabilities for 300, 600 and 1000 hours (as computed by (1)) are $0.1175,0.3173$ and 0.5500 respectively, while the results obtained by the stochastic approach are $0.1153,0.3136$ and 0.5477 by using a sequence length of $10 \mathrm{k}$ bits. As shown in Fig. 6, the accuracy of the stochastic approach can be further improved with longer stochastic sequences. In general, the stochastic approach accurately computes the failure probability at a reasonable sequence length (e.g. 10k bits). 


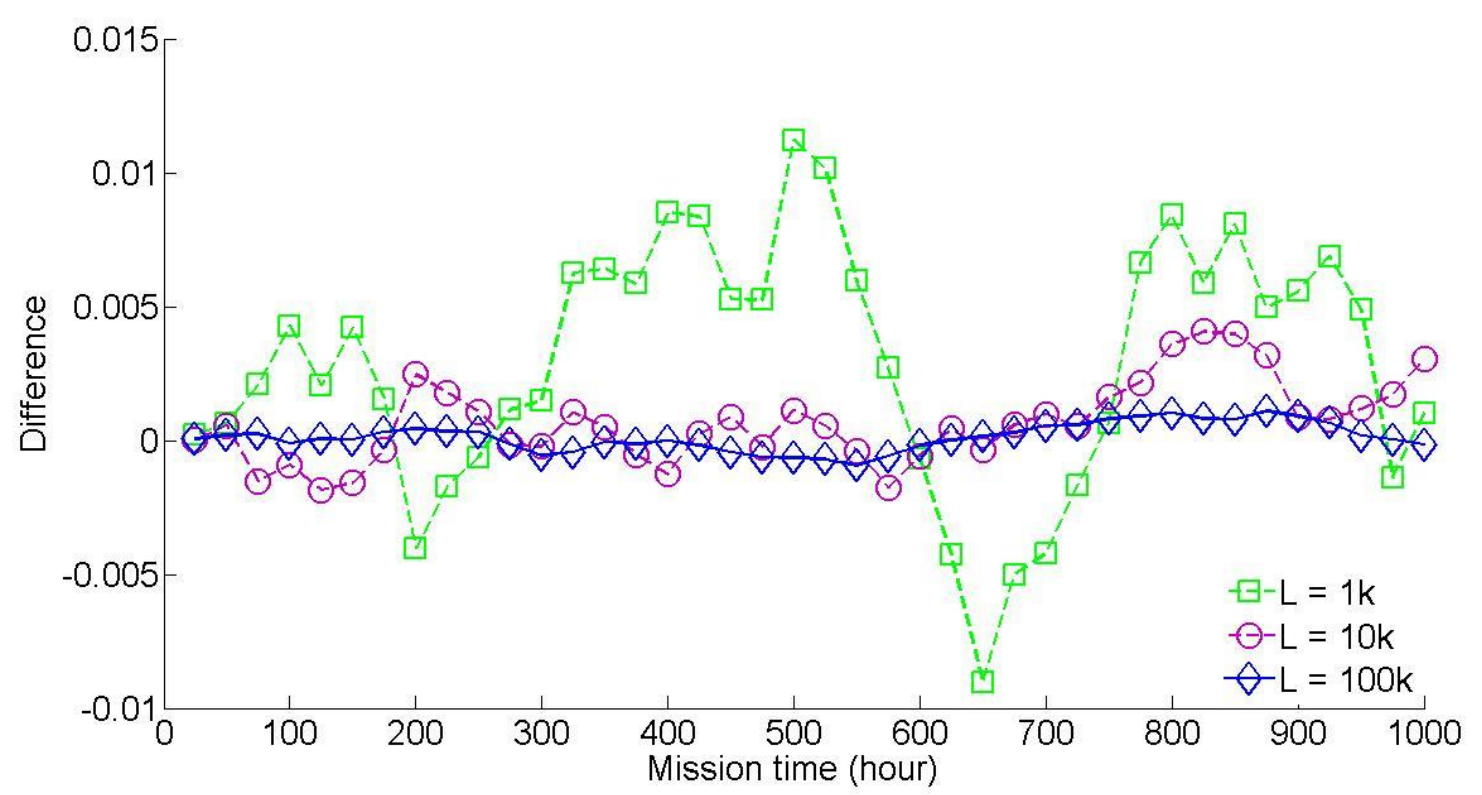

Fig. 6 The differences in the failure probabilities obtained by the stochastic approach and an accurate analysis for the WSP in Fig. 1.

\section{Stochastic models for CCFs and majority voters}

The stochastic model for common cause failure (CCF) is presented next for analyzing a general DFT. This is followed by an improved model that considers the CCF's probabilistic behavior, i.e., probabilistic CCFs (PCCFs). Finally, a stochastic majority voter is proposed.

\section{1) Stochastic model for CCFs}

Generally, CCFs are usually modeled by two types of methods: explicit methods [33, 34] and implicit methods $[35,36]$. In this paper, an explicit method is modeled by a stochastic approach and the CCF is considered as a basic event. To model dependent CCFs, a multiplexer is used with the stochastic sequences as inputs, as shown in Fig. 7(a). For a DFT [37], a hurricane occurs with a probability of $p(h)=0.015$. As floods usually occur in conjunction with hurricanes, the dependent relationship between the hurricane and flood can be described by conditional probabilities. The occurrence of floods is usually conditional on the occurrence of hurricanes, denoted as $p(b=f \mid h)=0.55$ and $p(a=f \mid \bar{h})=0.035$. These conditional probabilities can be 
derived from available weather information [38].

The function computed by the 2-to-1 multiplexer in Fig. 7 (a) is given by:

$$
p(f)=p(a=f \mid \bar{h}) \cdot p(\bar{h})+p(b=f \mid h) \cdot p(h),
$$

where $f \mid \bar{h}$ and $f \mid h$ are the events of floods $(f)$ conditional on the occurrence of a hurricane $(h)$. The output of the multiplexer is determined by the value of the control bit. For the 2-to-1 multiplexer of Fig. 7(a), one of the inputs is selected as the output according to the distributions of 0 s and 1s in the control sequence encoding the signal probability of $h$. For a sequence length of 10k bits, the input sequences for probabilities of $p(f \mid h)=0.55$ and $p(f \mid \bar{h})=0.035$ consist of 5500 and $3501 \mathrm{~s}$, respectively. If the random input sequences are independent, the output of the multiplexer is expected to be 0.0427 (by (5)), i.e. approximately 427 1s are expected in the output sequence for a sequence length of $10 \mathrm{k}$ bits. If multiple conditions are considered, for example to compute $p(A B C)$ based on $p(A B)$ and $p(C \mid A B)$, two conditions can first be combined, e.g., using an AND gate for a conjunction of the two events $A$ and $B$; then this new condition can be used as the control input to a multiplexer for computing the joint probability $p(A B C)$. This process is shown in Fig. 7(b). The computed result is however approximate due to the inevitable stochastic fluctuations inherent in the processing of the random binary bit streams. This is an important feature in stochastic computation as probabilistic values are propagated rather than deterministic ones. 


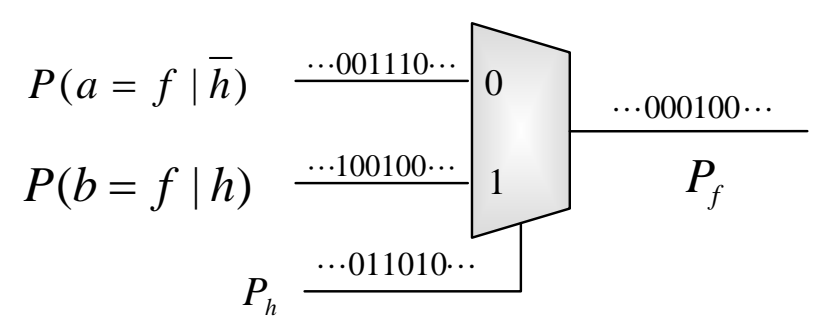

(a)

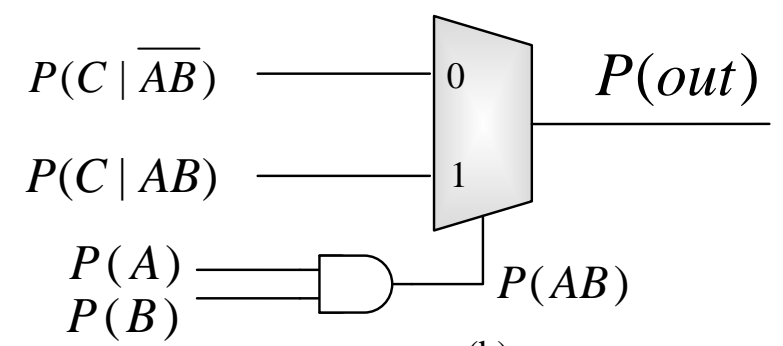

(b)

Fig. 7 (a) A stochastic multiplexer model for the dependency relationship between the two dependent CCFs of flood $(f)$ and hurricane $(h)$, and (b) A stochastic model for computing the joint probabilities of multiple conditions.

It has been shown in [18] that when the initial probabilities are encoded by non-Bernoulli sequences, the stochastic fluctuations are significantly reduced compared with the use of Bernoulli sequences. For the previous example, the occurrence probability of floods is obtained by using a multiplexer with stochastic non-Bernoulli sequences; the mean and variance are reported in Table 2 for a number of simulations using different sequence lengths. As shown by the simulation results, the evaluation accuracy is better for the stochastic approach with a smaller variance and it can be improved with an increase of sequence length.

Table 2 Mean and variance of the occurrence probability of flood obtained by using the stochastic approach and Monte Carlo (MC) method for 1,000 experiments with different sequence lengths or simulation runs.

\begin{tabular}{|c|c|c|c|c|}
\hline \multicolumn{2}{|c|}{$\begin{array}{l}\text { Sequence length } L \text { (bits) / Simulation runs } \\
\qquad N\end{array}$} & $N / L=1 \mathrm{k}$ & $N / L=10 \mathrm{k}$ & $N / L=100 \mathrm{k}$ \\
\hline \multirow[t]{2}{*}{ Stochastic approach } & Mean & 0.04264 & 0.04274 & 0.04274 \\
\hline & Variance & $3.892 \times 10^{-6}$ & $4.159 \times 10^{-7}$ & $3.829 \times 10^{-8}$ \\
\hline \multirow{2}{*}{$\begin{array}{l}\text { Monte Carlo } \\
\text { simulation }\end{array}$} & Mean & 0.04284 & 0.04265 & 0.04271 \\
\hline & Variance & $5.5956 \times 10^{-5}$ & $4.1978 \times 10^{-6}$ & $4.0124 \times 10^{-7}$ \\
\hline
\end{tabular}

The stochastic approach efficiently computes the occurrence probability of dependent CCFs as evidenced by the average run time in Table 2. Moreover, the variance is significantly 
reduced with an increase of sequence length. The use of a sequence length of $1 \mathrm{k}$ bits generates very accurate results, with a relative disparity (RD) of approximately $0.23 \%$, compared to the analytical result of 0.0427 computed by (5). RD is defined as:

$$
R D=\left(p-p_{0}\right) / p_{0}
$$

where $p$ and $p_{0}$ are the probabilities obtained by using the stochastic approach and an accurate analysis, respectively. For an increased sequence length, a smaller RD can be obtained by the stochastic approach.

\section{2) A stochastic model for PCCF}

A mechanical system can be subject to multiple CCFs, as denoted by $C C F_{1}, C C F_{2}, \cdots, C C F_{m}$. The failure of a dependent event affected by a specific CCF (say $C C F_{i}$ ) occurs with certain probability, so the CCF is considered as a probabilistic CCF (PCCF). The occurrence probability of a PCCF is given by $\gamma_{i}=p\left(\right.$ Dependent event fails $\mid C C F_{i}$ occurs $) ; \gamma_{i}$ may vary for different components affected by a CCF.

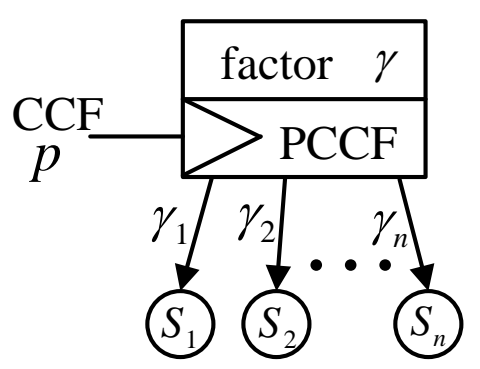

(a)

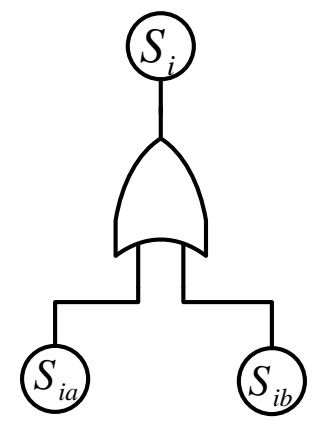

(b)

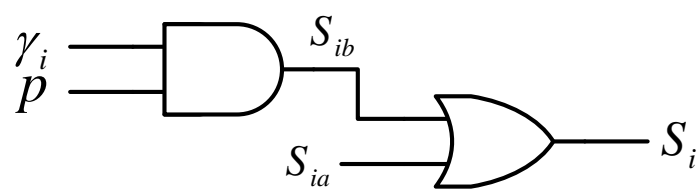

(c)

Fig. 8 (a) A PCCF gate [25], (b) a combinational model for the PCCF gate, and (c) proposed stochastic model for the PCCF gate.

In Fig. 8(a), the CCF occurs as a trigger event with probability $p$; then one or more dependent events affected by the trigger event fail at a specific probability. For example, an event $S_{i}$ occurs 
with probability $\gamma_{i}$ if the trigger event occurs. Let $S_{i a}$ and $S_{i b}$ denote the failures of the basic event $S_{i}$ without and with considering the effect of a CCF; then if $S_{i a}$ or $S_{i b}$ occurs, the output event $S_{i}$ fails. Thus, the failure of the event $S_{i}$ can be modeled as an OR gate with two input events $S_{i a}$ and $S_{i b}$ (as illustrated in Fig. 8(b)). The event $S_{i b}$ occurs with probability $p \cdot \gamma_{i}$, where $p$ is the occurrence probability of the $\mathrm{CCF}$ and $\gamma_{i}$ is the conditional failure probability of the dependent event $S_{i}$ affected by the CCF. A stochastic model is proposed to implement the PCCF, as shown in Fig. 8(c). The simulation results for this model are shown in Table 3 for $p(A)=0.1$ and $p(C C F)=0.01$; the probability of event $A$ affected by the CCF is given by $\gamma=0.3$.

Table 3 Mean and variance of the simulated occurrence probability of a component $A$ under a PCCF by applying the stochastic approach for 1,000 simulations. The average run time is also provided.

\begin{tabular}{c|c|c|c|c}
\hline \hline \multicolumn{2}{c|}{ Sequence length $L($ bits $)$} & $L=1 \mathrm{k}$ & $L=10 \mathrm{k}$ & $L=100 \mathrm{k}$ \\
\hline \multirow{2}{*}{$p(A)$} & Mean & 0.1026 & 0.1027 & 0.1027 \\
\cline { 2 - 5 } & Variance & $1.9439 \times 10^{-6}$ & $2.1275 \times 10^{-7}$ & $1.9579 \times 10^{-8}$ \\
\hline \multicolumn{2}{c}{ Average run time (s) } & 0.000642 & 0.006680 & 0.071408 \\
\hline
\end{tabular}

As revealed in Table 3, the proposed stochastic approach accurately computes the occurrence probability of PCCFs. The relative disparity (given by (6)) is approximately $0.0974 \%$ for a sequence length of 1,000 bits compared with the analytical result of 0.1027 [25]. Furthermore, the variance can be significantly reduced with a longer sequence length, thus the accuracy in the failure probability obtained by the stochastic approach increases with an increase of sequence length.

\section{3) A stochastic model for Majority voter}

The stochastic structure for a majority voter (2/3) is shown in Fig. 9, as implemented by stochastic logic in Fig. 9(b). The analytical expression for the output probability of the majority voter $(2 / 3)$ is given by [40]: 


$$
p(\text { Out })=p(A) \cdot p(B)+p(A) \cdot p(C)+p(B) \cdot p(C)-2 \cdot p(A) \cdot p(B) \cdot p(C)
$$

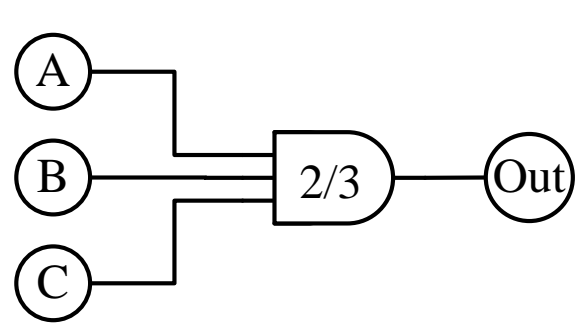

(a)

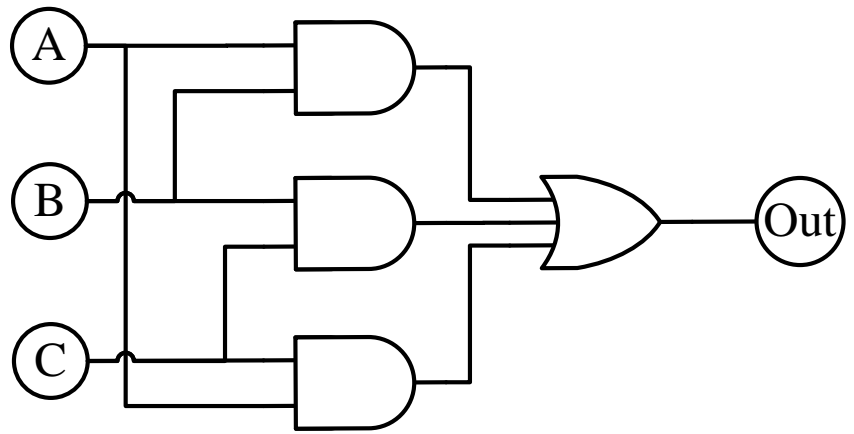

(b)

Fig. 9 (a) A majority voter (2/3) [40]; (b) A stochastic model for the majority voter (2/3).

Table 4 Mean and variance of the failure probabilities of $2 / 3$ and $3 / 5$ majority voters, obtained by the stochastic approach. The average simulation time is also provided.

For $2 / 3$ voter, $p(A)=0.3, p(B)=0.6, p(C)=0.2$

\begin{tabular}{c|c|c|c}
\hline Sequence length L (bits) & $L=1 \mathrm{k}$ & $L=10 \mathrm{k}$ & $L=100 \mathrm{k}$ \\
\hline Mean & 0.2882 & 0.2880 & 0.2880 \\
\hline Variance & $6.3098 \times 10^{-5}$ & $5.4278 \times 10^{-6}$ & $5.8191 \times 10^{-7}$ \\
\hline Average simulation time (s) & 0.002396 & 0.002264 & 0.013988 \\
\hline
\end{tabular}

For $3 / 5$ voter, $p(A)=0.2, p(B)=0.4, p(C)=0.5, p(D)=0.1, p(E)=0.4$

\begin{tabular}{c|c|c|c}
\hline \hline Sequence length $L$ (bits) & $L=1 \mathrm{k}$ & $L=10 \mathrm{k}$ & $L=100 \mathrm{k}$ \\
\hline Mean & 0.1783 & 0.1781 & 0.1780 \\
\hline Variance & $6.6767 \times 10^{-5}$ & $6.2741 \times 10^{-6}$ & $6.3871 \times 10^{-7}$ \\
\hline Average simulation time (s) & 0.003154 & 0.027721 & 0.281911 \\
\hline \hline
\end{tabular}

For a (2/3) majority voter with inputs' failure probabilities given in Table 4, the output failure probability is 0.2880 by (7); the relative disparity (RD) is $0.069 \%$ (given by (6)) for the stochastic approach using sequences of $1 \mathrm{k}$ bits. For a (3/5) majority voter, the output probability is 0.1780 using the analysis of [40], while the RD is approximately $0.17 \%$ for the stochastic approach with 
$L=1 \mathrm{k}$ bits. Thus, the error in the failure probability for the stochastic approach decreases with the increase of sequence length. Similar stochastic circuits can be constructed for majority gates with more than three inputs.

\section{DFT analysis flow}

Following the proposed stochastic models for the spare and PAND gates [20], the process for evaluating the top event's failure probability of a general DFT with PCCFs, consists of the following steps:

i. $\quad$ Replace the original spare gate with the proposed stochastic model for WSP/CSP in Fig. 5(b);

ii. Substitute the original FDEP and PAND gates with the OR model [21, 22] and the stochastic PAND model in [20] respectively; then a DFT with dynamic gates can be implemented by combinational logic;

iii. Encode the events' failure probabilities at different time steps into non-Bernoulli sequences;

iv. If PCCFs are considered in the DFT, an additional PCCF module is required for each of the basic events subject to PCCFs. Moreover, if the CCFs are dependent, a stochastic multiplexer is used to model the effect of the dependency.

v. Derive the top event's failure probability at different time steps by propagating the nonBernoulli sequences through the stochastic models.

\section{CASE STUDIES}

In this section, several case studies are presented to show the efficiency and accuracy of the stochastic method, in comparison with the analytical method of [10] and the Monte Carlo (MC) approach of [15]. Simulations are performed for DFTs with and without probabilistic common cause failures (PCCFs). Furthermore, the effect of dependent PCCFs is also analyzed. Nonexponential distributions of the basic events are also considered to show the capabilities of the 
stochastic approach to handle general cases. All simulations are run on a computer with a 3.10 GHz i3-2100 microprocessor and a 6 GB memory.

Let the failure probability of a basic event $B$ at time $i$ be $F_{i}^{B}, i \in\{1,2, \cdots, M\}$, obtained as the failure $c d f$ for the basic event; then the failure probability of the DFT is given by:

$$
F(i)=f\left(F_{i}^{B}\right)
$$

where $f($.$) indicates the logic operation determined by the system's topology. Hence, the failure$ probability vector for the entire mission time is given by a vector $\boldsymbol{F}=(F(1), F(2), \cdots, F(M))$, where $M$ indicates the number of discretized intervals of the mission time. The failure probability vectors obtained using the stochastic, analytical [10] and MC [15] approaches are then represented by $\boldsymbol{F}_{S}, \boldsymbol{F}_{A}$ and $\boldsymbol{F}_{M C}$ respectively. Hence, $\Delta \boldsymbol{F}_{S-A}$ indicates the disparity vector for the stochastic and analytical methods; $\Delta \boldsymbol{F}_{M C-A}$ represents the disparity vector for the MC and analytical approaches. Similarly, the norms, $\|\cdot\|_{1},\|\cdot\|_{2}$ and $\|\cdot\|_{\infty}$, are used to measure the differences of these failure probability vectors.

\section{A. HECS with and without PCCFs}

A DFT of the Hypothetical Example Computer System (HECS) (from [26] and shown in Fig. 10) is used to illustrate the efficiency and accuracy of the proposed stochastic method.

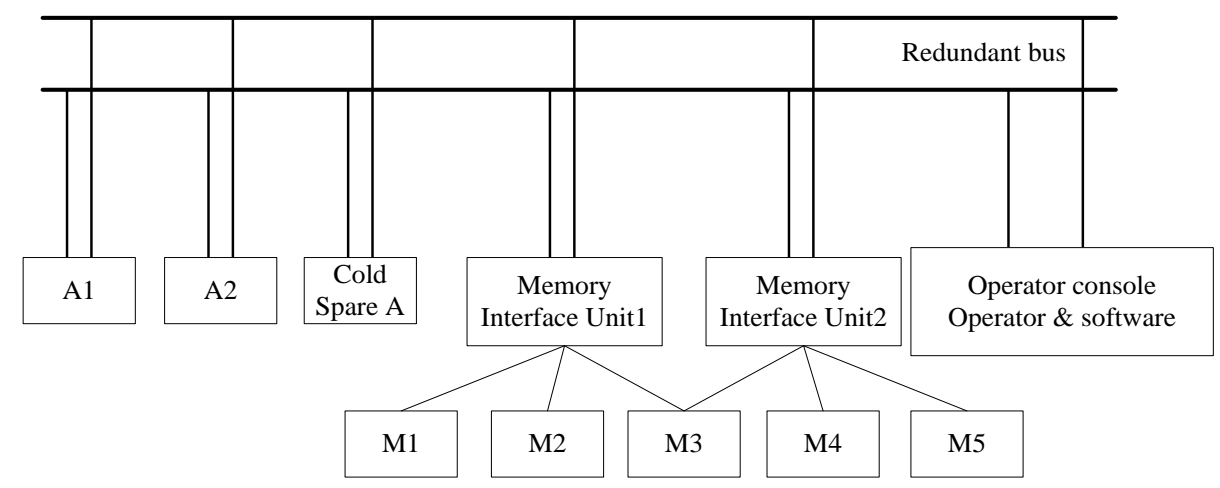

Fig. 10 The Hypothetical Example Computer System (HECS) [26]. 
The correct operation of the HECS is determined by the states of different systems such as the processor (A1, A2 and A), memory, bus and application interfaces [41]. The HECS will fail if any of the four subsystems fail. The computer of Fig. 10 is modeled by a DFT in Fig. 11(a) as illustrated in [41]. Using the stochastic models of the dynamic gates, a complete stochastic system can be constructed for the HECS, as shown in Fig. 11(b).

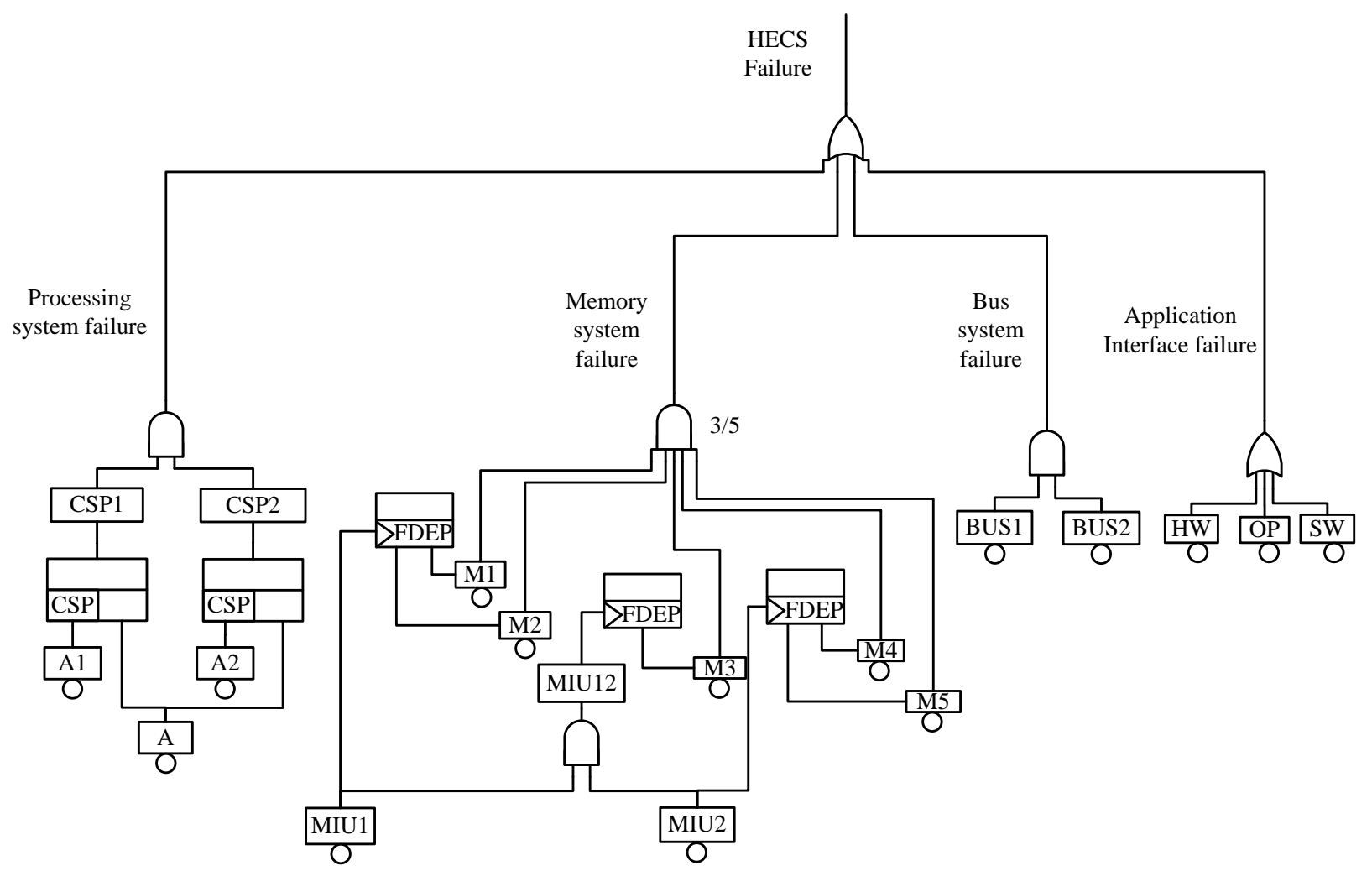

(a) 


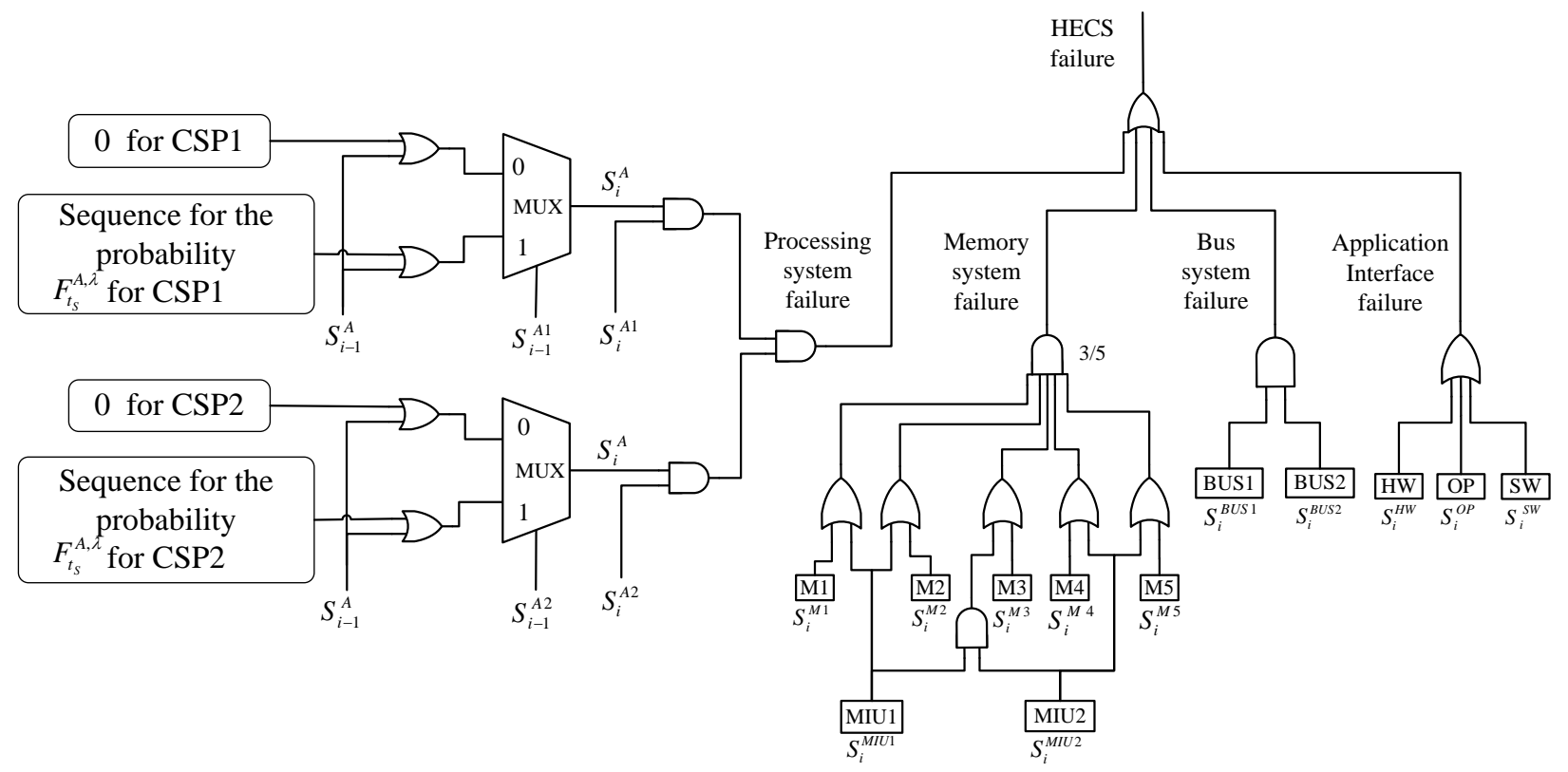

(b)

Fig. 11 (a) A DFT of HECS with CSP, FDEP and static gates [41], and (b) the stochastic model for the HECS with constituent dynamic gate models.

Table 5 The failure rates of the basic events in the HECS [26, 41].

\begin{tabular}{c|c}
\hline \hline Basic event & Failure rate $\left(h^{-1}\right)$ \\
\hline A1 A2 A & $10^{-4}$ \\
\hline M1M2 M3 M4 M5 & $6 \times 10^{-5}$ \\
\hline MIU1,MIU2 & $5 \times 10^{-5}$ \\
\hline BUS1,BUS2 & $10^{-6}$ \\
\hline HW & $5 \times 10^{-5}$ \\
\hline SW & $3 \times 10^{-2}$ \\
\hline OP & $10^{-3}$ \\
\hline \hline
\end{tabular}


The mission time of the HECS is assumed to be 100 hours and the failure behaviors of the basic events in the HECS are considered to be exponentially distributed (the failure rates are shown in Table $5[26,41])$.

For a mission time of 100 hours, the difference in failure probabilities of the HECS and the average simulation time are shown in Table 6 for the different approaches. $N$ and $L$ denote the number of simulation runs for the MC method and the sequence length for the stochastic approach, respectively. The norms of the disparity vectors are presented for the stochastic and MC [15] approaches.

Table 6 Norms of the differences in the top event's failure probability vectors obtained by the proposed stochastic approach and MC simulation for the DFT in Example A. The average run time is also provided.

\begin{tabular}{|c|c|c|c|c|}
\hline & & $N / L=1 \mathrm{k}$ & $N / L=10 \mathrm{k}$ & $N / L=100 \mathrm{k}$ \\
\hline \multicolumn{2}{|c|}{$\left\|\Delta \boldsymbol{F}_{S-A}\right\|_{1}$} & 0.1749 & 0.0529 & 0.0168 \\
\hline \multicolumn{2}{|c|}{$\left\|\Delta \boldsymbol{F}_{S-A}\right\|_{2}$} & 0.0225 & 0.0066 & 0.0023 \\
\hline \multicolumn{2}{|c|}{$\left\|\Delta \boldsymbol{F}_{S-A}\right\|_{\infty}$} & 0.0064 & 0.0016 & $7.0872 \times 10^{-4}$ \\
\hline \multicolumn{2}{|c|}{$\left\|\Delta \boldsymbol{F}_{M C-A}\right\|_{1}$} & 0.8199 & 0.2830 & 0.1024 \\
\hline \multicolumn{2}{|c|}{$\left\|\Delta \boldsymbol{F}_{M C-A}\right\|_{2}$} & 0.1079 & 0.0386 & 0.0140 \\
\hline \multicolumn{2}{|c|}{$\left\|\Delta \boldsymbol{F}_{M C-A}\right\|_{\infty}$} & 0.0364 & 0.0134 & 0.0049 \\
\hline \multirow{3}{*}{$\begin{array}{l}\text { Average run } \\
\text { time (s) }\end{array}$} & Accurate Analysis & \multicolumn{3}{|c|}{0.001738} \\
\hline & Stochastic & 0.0509 & 0.3868 & 3.9639 \\
\hline & $\mathrm{MC}$ & 0.1414 & 1.2583 & 12.596 \\
\hline
\end{tabular}

As shown in Table 6, the proposed stochastic approach requires a shorter run time and results in a smaller variance in the computed failure probability; hence, it is more efficient and more accurate than the MC method. The evaluation accuracy can be further improved by increasing the 
sequence length $L$. However, a trade-off between precision and efficiency must be determined when selecting the sequence length. Although the accurate analysis results in the shortest run time, the significantly longer time required for deriving the analytical expressions is not included in the value reported in Table 6 .

In practice, the failure distribution is not limited to an exponential distribution if other factors such as aging are taken into consideration. Therefore, a non-exponential distribution may be required for a more accurate modeling. A Weibull distribution is considered for a DFT with nonexponentially distributed basic events. The $p d f$ and $c d f$ of a Weibull distribution are given by

$$
f(t)=\frac{k}{\lambda}\left(\frac{t}{\lambda}\right)^{k-1} e^{-(t / \lambda)^{k}}
$$

and

$$
F(t)=1-e^{-(t / \lambda)^{k}}
$$

respectively, where $k$ and $\lambda$ are the shape and scale parameters respectively.

Assume that the basic events A1 and BUS1 follow a Weibull distribution with $\lambda=2$ and $k=$ 0.1, while the failures of the other basic events are exponentially distributed. Furthermore, assume that BUS1 and HW are subject to a CCF (i.e. $\gamma_{i}=1$ ) with an occurrence probability of 0.1. Fig. 12 reveals the difference of the top event's failure probabilities for a mission time of 100 hours, obtained by both the stochastic approach and MC simulation [15]. It can be seen that the difference between the stochastic and MC approach decreases with the increase of the sequence length. For a sequence length (or simulation runs) of $10 \mathrm{k}$ bits, $\|\cdot\|_{1} \cdot\|\cdot\|_{2},\|\cdot\|_{\infty}$ of the differences in the failure probability vectors obtained by the two approaches are $0.3087,0.0387$ and 0.0133 respectively. As revealed by these norm values, a DFT with non-exponentially distributed basic events subject to PCCFs can be efficiently evaluated by the proposed stochastic approach with a reasonable sequence length. 


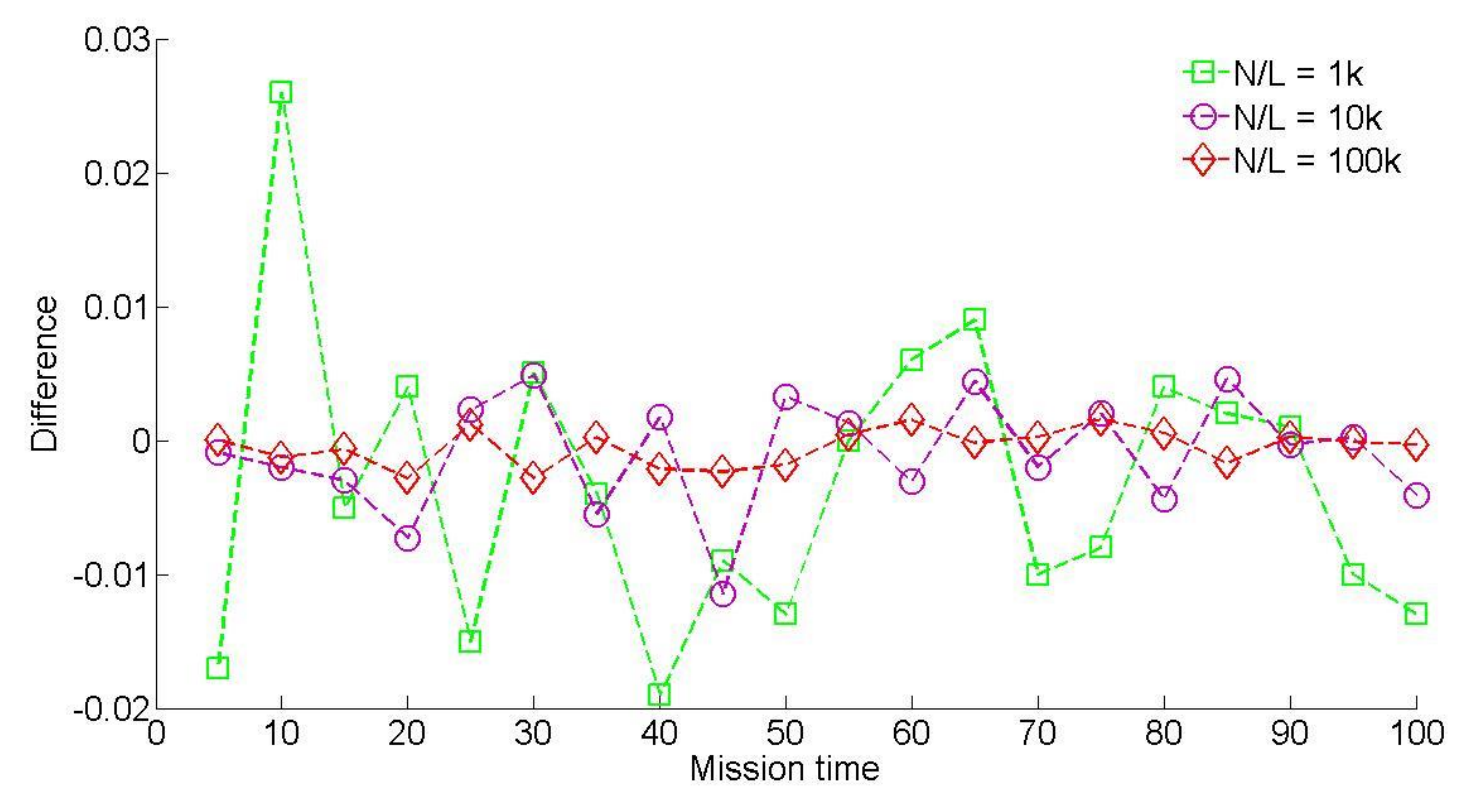

Fig. 12 Difference in the failure probabilities of the top event for the HECS for a mission time of 100 hours.

\section{B. DFT with dependent PCCFs}

A DFT with WSP, FDEP and PAND gates is analyzed next to show the efficiency of the stochastic approach (Fig. 13(a)). Assume that $h, l$ and $f$ denote the events of hurricanes, lightning strikes and floods respectively. Table 7 shows the exponentially distributed failure rates of the components; non-exponential distributions will be dealt with subsequently. The occurrence probabilities of a hurricane and a lightning strike are given as $p(h)=0.015$ and $p(l)=0.025$ respectively. The dependencies between the CCFs are given as the conditional probabilities between a hurricane and floods, i.e., $p(f \mid h)=0.55$ and $p(f \mid \bar{h})=0.035$ as obtained from weather information [38]. The probability of a component affected by a CCF is assumed to be $\gamma_{i}=0.8$ for $i=1,2,3$ (where $i$ indicates a different CCF, i.e., $h, l, f$, respectively). For this DFT, a stochastic model is constructed in Fig. 13(b) with the PAND model of [20] and the stochastic models in Figs. 7 and 8 for considering the effects of PCCFs. 
Table 7 Component failure rates $\left(10^{-3} /\right.$ hour $)$.

\begin{tabular}{c|c|c|c}
\hline \hline Basic events & Failure rates & Basic events & Failure rates \\
\hline $\mathrm{A}$ & 1.5 & $\mathrm{~B}$ & 1.0 \\
\hline $\mathrm{C}$ & 4.0 & $\mathrm{D}$ & 1.0 \\
\hline $\mathrm{E}$ & 2.0 & $\mathrm{~F}$ & 1.0 \\
\hline $\mathrm{G}$ & 3.0 & $\mathrm{H}$ & 2.0 \\
\hline $\mathrm{I}$ (spare) & 1.0 & I (working) & 2.0 \\
\hline \hline
\end{tabular}

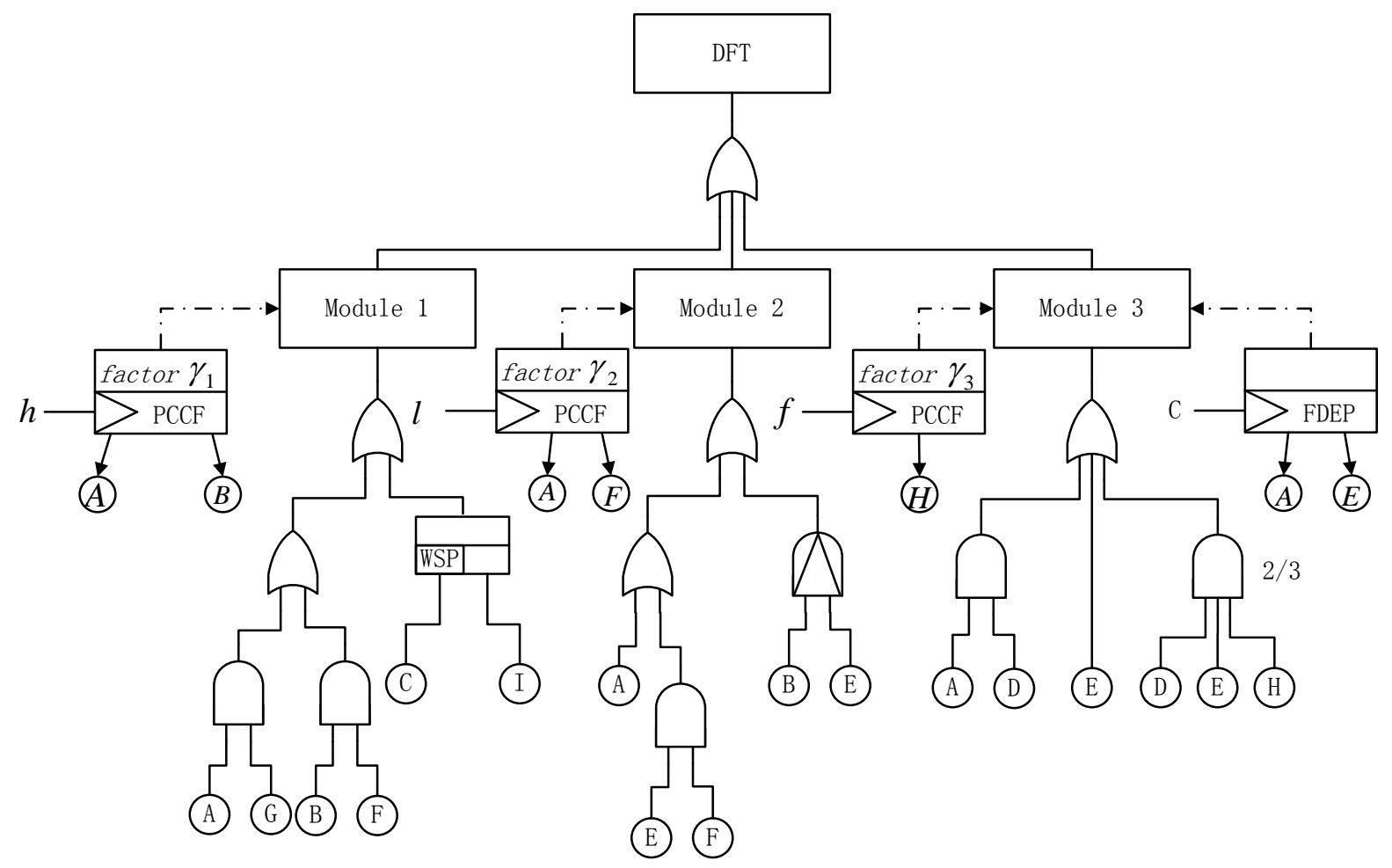

(a) 


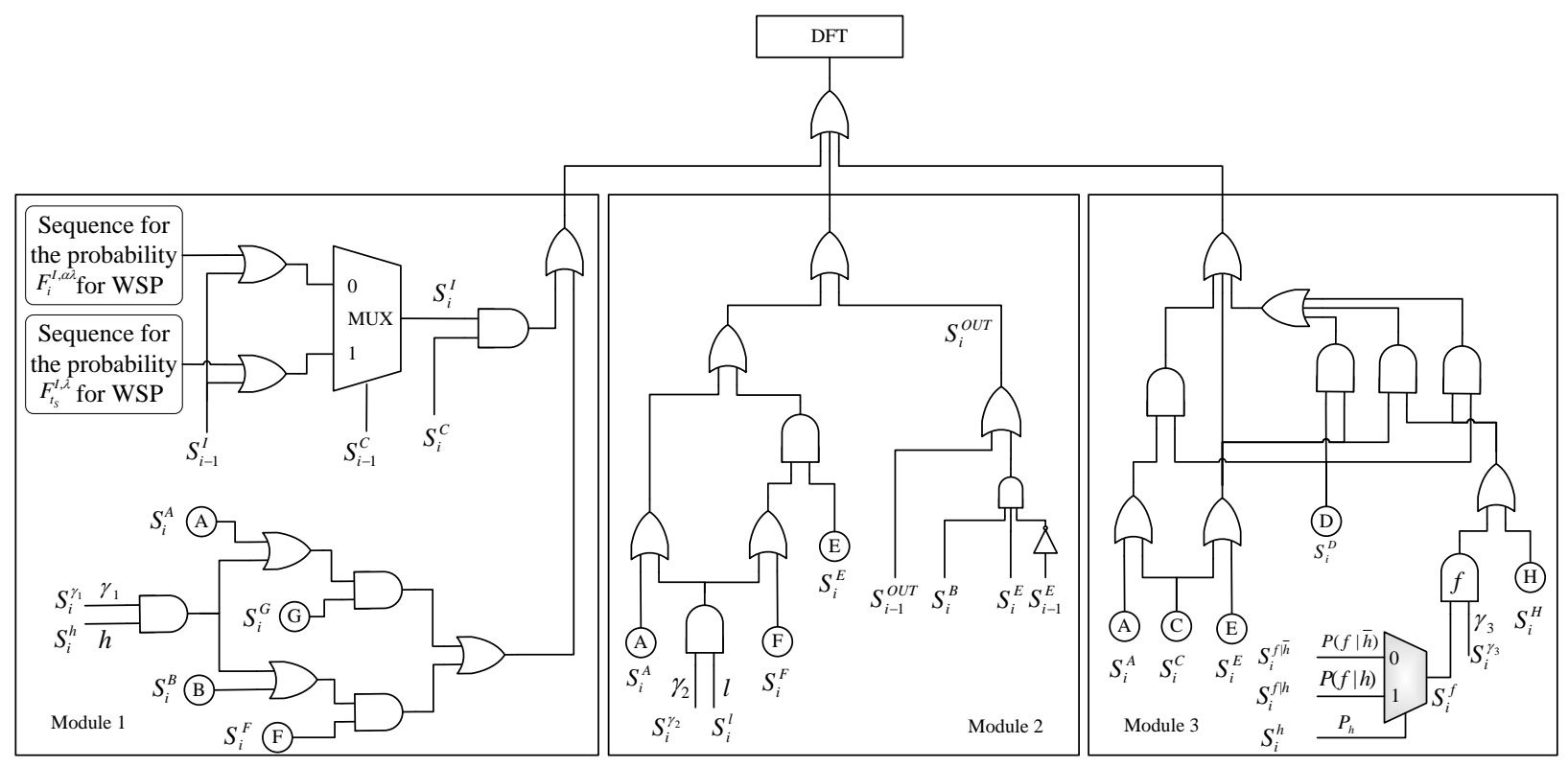

(b)

Fig. 13 (a) A DFT with dependent PCCFs (taken from [24]), and (b) a stochastic model for the DFT in (a).

The average run time and norms of the differences in the failure probability vectors of the DFT obtained by the stochastic and MC [15] approaches are given in Table 8 for a mission time of 200 hours. Also shown are the failure probabilities by considering PCCFs for each of the modules. 
Table 8 Norms of the differences in the top event's failure probability vectors of the DFT in Example B, and the average run time for the proposed stochastic approach and MC simulation in [15].

\begin{tabular}{|c|c|c|c|c|}
\hline \multicolumn{2}{|c|}{ Under CCFs (i.e. $\gamma_{i}=1$ ) } & $N / L=1 \mathrm{k}$ & $N / L=10 \mathrm{k}$ & $N / L=100 \mathrm{k}$ \\
\hline \multicolumn{2}{|c|}{$\left\|\Delta \boldsymbol{F}_{S-M C}\right\|_{1}$} & 2.4370 & 0.7457 & 0.2301 \\
\hline \multicolumn{2}{|c|}{$\left\|\Delta \boldsymbol{F}_{S-M C}\right\|_{2}$} & 0.2227 & 0.0680 & 0.0205 \\
\hline \multicolumn{2}{|c|}{$\left\|\Delta \boldsymbol{F}_{S-M C}\right\|_{\infty}$} & 0.0460 & 0.0148 & 0.0041 \\
\hline \multirow{3}{*}{$\begin{array}{l}\text { Average run time } \\
\text { (s) }\end{array}$} & Accurate Analysis & \multicolumn{3}{|c|}{0.0049} \\
\hline & Stochastic & 0.2207 & 2.0595 & 16.282 \\
\hline & $\mathrm{MC}$ & 0.3825 & 3.5453 & 36.664 \\
\hline \multicolumn{2}{|c|}{ Under PCCFs with $\gamma_{i}=0.8$} & $N / L=1 \mathrm{k}$ & $N / L=10 \mathrm{k}$ & $N / L=100 \mathrm{k}$ \\
\hline \multicolumn{2}{|c|}{$\left\|\Delta \boldsymbol{F}_{S-M C}\right\|_{1}$} & 2.5580 & 0.8148 & 0.2498 \\
\hline \multicolumn{2}{|c|}{$\left\|\Delta \boldsymbol{F}_{S-M C}\right\|_{2}$} & 0.2324 & 0.0722 & 0.0216 \\
\hline \multicolumn{2}{|c|}{$\left\|\Delta \boldsymbol{F}_{S-M C}\right\|_{\infty}$} & 0.0450 & 0.0152 & 0.0042 \\
\hline \multirow{3}{*}{$\begin{array}{l}\text { Average run time } \\
\text { (s) }\end{array}$} & Accurate Analysis & \multicolumn{3}{|c|}{0.0061} \\
\hline & Stochastic & 0.2445 & 1.8734 & 21.031 \\
\hline & $\mathrm{MC}$ & 0.4783 & 4.9198 & 41.159 \\
\hline
\end{tabular}

In Monte Carlo simulation, the result follows approximately a Gaussian distribution for a large number of runs; this is also applicable to stochastic computation [18]. In this case, a parameter $z_{c}$ can be used to determine the confidence interval of the simulated results [39]. The error in the computed result is then given by

$$
E=\frac{\mathrm{z}_{\mathrm{c}}}{\mu} \sqrt{\frac{v}{m}}
$$

where $\mu$ and $v$ are the accurate mean and variance of the distribution of the results, and $m$ is the number of simulations (or equivalently, the number of bits in a stochastic sequence). For a confidence level of $95 \%, z_{c}=1.96$. For the failure rates in Table 7 and $\gamma_{i}=0.8$, stochastic 
sequences of one million bits are used to find the accurate mean and variance, as 0.8119 and 0.1527 , respectively, for the stochastic approach. For a sequence length of $10 \mathrm{k}$, the error is then obtained by (11) as $0.9434 \%$ (i.e. less than $1 \%$ ) at a confidence level of $95 \%$. As per (11), the error decreases with an increase of sequence length at a given confidence level. The required sequence length can thus be estimated by (11) for achieving a desired evaluation accuracy.

For this DFT, the top event's failure probability for a mission time of 200 hours is plotted in Fig. 14 for two values of $\gamma_{i}$, where $\gamma_{i}, i=1,2,3$, indicates the probability that the basic event is affected by a CCF. In Fig. 14(a), the failures of the basic events are assumed to be exponentially distributed; for $\gamma_{i}=0$, i.e., when the effect of a CCF is not considered, the failure probability is underestimated compared to the case when the occurrence of a CCF will definitely cause a failure of the basic event (i.e. when $\gamma_{i}=1$ ). In the latter case, the failure probability is overestimated compared to the case when the probabilistic behavior of a CCF is considered, i.e., when $0<\gamma_{i}<$ 1 (for which the failure probabilities would lie between the values shown in Fig. 14(a)). Furthermore, assume that components $\mathrm{A}$ and $\mathrm{D}$ follow a Weibull distribution with $\lambda=2$ and $k=$ 0.1, while the failure rates of the other basic events remain at the values given in Table 7. Fig. 14(b) plots the differences between the failure probabilities obtained by the stochastic and Monte Carlo approaches; the difference decreases with an increase of stochastic sequence length or the number of simulation runs. For a sequence length (or simulation runs) of 10k bits, the norms, $\|\cdot\|_{1} \cdot\|\cdot\|_{2}$, and $\|\cdot\|_{\infty}$, of the differences in the failure probability vectors of the stochastic and MC approaches are obtained as $0.8198,0.0734$ and 0.0136 , respectively. 


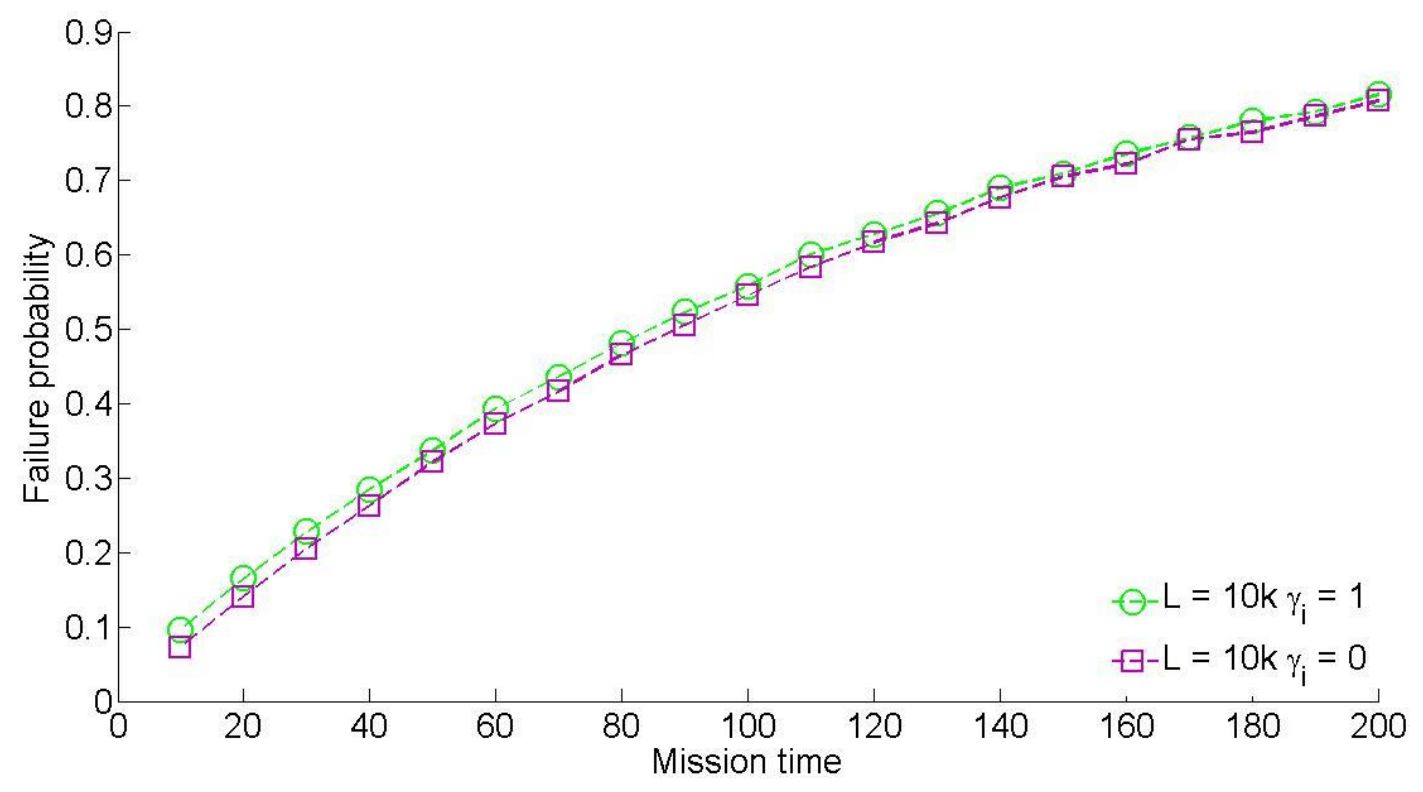

(a)

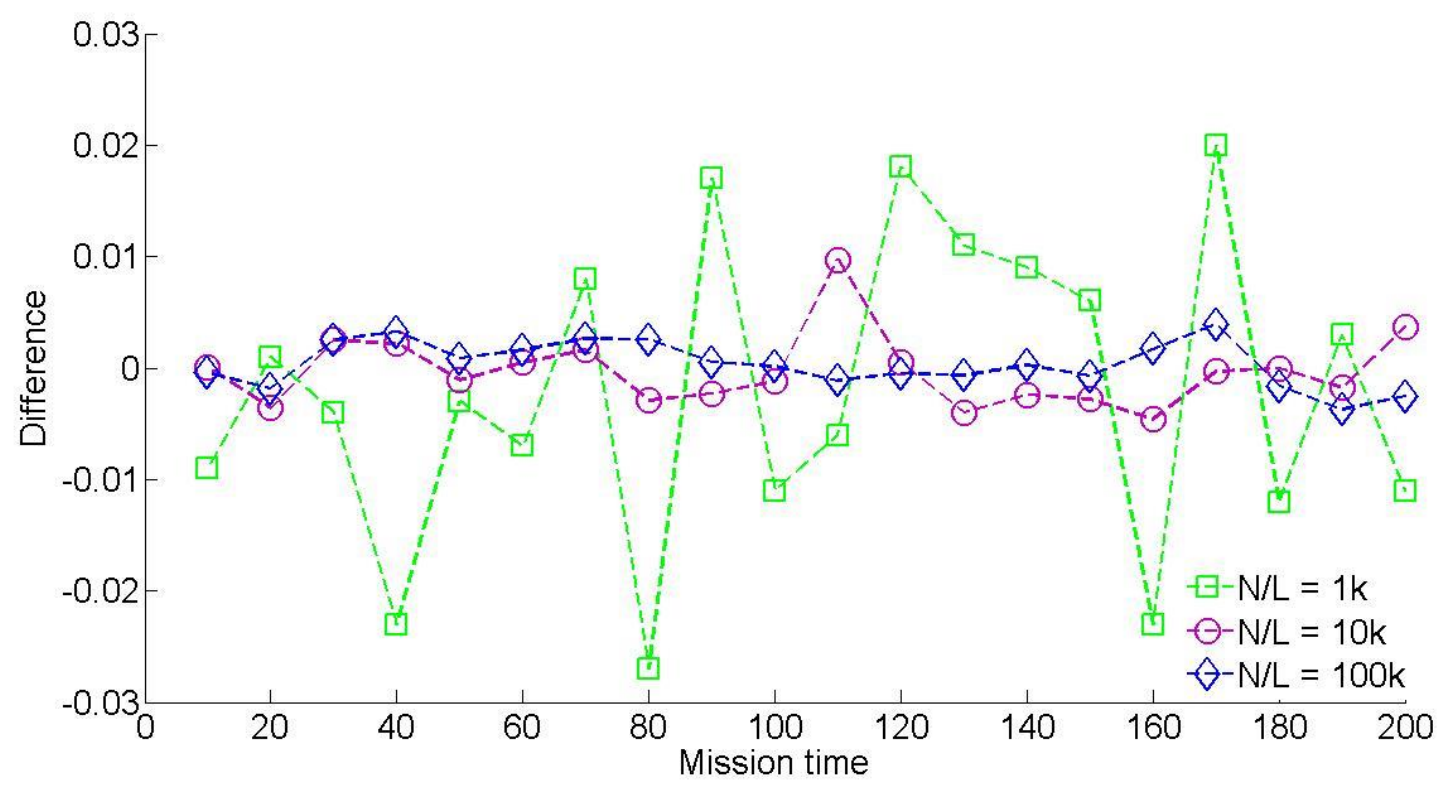

(b)

Fig. 14 Example B: (a) Failure probability of the DFT subject to PCCFs for $\gamma_{i}=0$ and $\gamma_{i}=1$ (for basic events with exponentially distributed failures, using a sequence length of 10k bits), and (b) Difference of the failure 
probabilities of the DFT subject to PCCFs for $\gamma_{i}=0.8$ (for basic events with non-exponentially distributed failures).

As can be seen from these results, the DFT systems (inclusive of the spare gate, PAND and FDEP gates) can be efficiently evaluated by the proposed stochastic approach. The stochastic approach is more efficient than a MC method [15] with an equivalent number of simulation runs. Furthermore, the accuracy of the proposed stochastic approach can be improved by increasing the sequence length. The required sequence length is determined as a trade-off between precision and efficiency. It is also shown that the reliability of a DFT system decreases by considering the effects of PCCFs that widely occur in practice. Hence, if the failure of certain component affected by PCCFs is not considered, the reliability of a DFT is likely to be overestimated. If the effect of PCCFs is considered to be deterministic in causing a failure, then the reliability of a DFT is underestimated.

\section{CONCLUSION}

In this paper, stochastic models have been proposed for analyzing a two-input spare gate and probabilistic common cause failures (PCCFs) in a dynamic fault tree (DFT); the WSP and CSP gates have been analyzed in detail. For a DFT with spare gates, a stochastic approach using the proposed models provides an efficient analysis of the DFT compared to an analytical approach. The use of non-Bernoulli sequences of random permutations of fixed numbers of $1 \mathrm{~s}$ and $0 \mathrm{~s}$ as initial input probabilities makes the stochastic approach more efficient and more accurate than Monte Carlo simulation. The effect of PCCF has been taken into consideration and a stochastic logic model has been constructed for dependent PCCFs. The efficiency and accuracy of the proposed stochastic approach have been shown by the case studies of several benchmark systems. 
Ongoing work includes the stochastic modeling of repair schemes and the assessment of multiple-valued DFT systems with imperfect fault coverage.

\section{REFERENCES}

[1] A. Clifton Ericson II.: Fault tree analysis - a history. In Proceedings of the 17th International System Safety Conference, August 16-21, 1999.

[2] N. G. .Leveson.: Safeware: System safety and computers.: Addison-Wesley, 1995.

[3] M. A. Boyd.: Dynamic fault tree models: techniques for analyses of advanced fault tolerant computer systems. Phd dissertation, Dept. of Computer Science, Duke University, 1991.

[4] J. B. Dugan, S. J. Bavuso, and M. A. Boyd.: Dynamic fault-tree models for fault-tolerant computer systems. IEEE Transactions on Reliability, 41(3):363-377, September 1992.

[5] G. Merle, J.-M. Roussel, J.-J. Lesage, A. Bobbio.: Probabilistic Algebraic Analysis of Fault Trees with Priority Dynamic Gates and Repeated Events. IEEE Trans. Reliability, vol. 59, no. 1, Mar. 2010, pp. 250-261.

[6] R. Manian, J. B. Dugan, D. Coppit, and K. J. Sullivan.: Combining various solution techniques for dynamic fault tree analysis of computer systems. In Proceedings of the 3rd IEEE International Symposium on High-Assurance Systems Engineering (HASE’98), USA, Nov. 1998, pp. 21-28.

[7] H. Boudali, P. Crouzen, and M. Stoelinga (2007).: Dynamic Fault Tree analysis through input/output interactive Markov chains. In Proceedings of the International Conference on Dependable Systems and Networks (DSN 2007), pp. 25-38.

[8] H. Boudali and J. B. Dugan.: A discrete-time Bayesian network reliability modeling and analysis framework. Reliability Engineering and System Safety, vol. 87, no. 3, pp. 337-349, 2005.

[9] T. Yuge, S.Yanagi.: Quantitative analysis of a fault tree with priority AND gates. Reliab Eng Syst Safety 2008; 93(11): 157783.

[10] S. Amari, G. Dill, E. Howald.: A new approach to solve dynamic fault trees. In: Annual IEEE reliability and maintainability symposium, 2003. p. 374-9.

[11] L. Xing.: An efficient binary-decision-diagram-based approach for network reliability and sensitivity analysis. IEEE Trans. Systems, Man, and Cybernetics, vol. 38, no. 1, pp. 105-115, Jan. 2007.

[12] O. Tannous, L. Xing, and J. B. Dugan.: Reliability Analysis of Warm Standby Systems using Sequential BDD. In Proc. of the 57th Annual Reliability \&Maintainability Symposium, FL, USA, 2011. 
[13] L. Xing, O. Tannous, and J. B. Dugan.: Reliability analysis of nonrepairable cold-standby systems using sequential binary decision diagrams. IEEE Trans. Syst., Man, Cybern. A, Syst., Humans, vol. 42, no. 3,pp. 715-726, May 2012.

[14] W. Long, T. L. Zhang, Y. F. Lu, and M. Oshima.: On the quantitative analysis of sequential failure logic using Monte Carlo method for different distributions. In Proc. Probabilistic Saf. Assessment Manage., 2002, pp. 391-396.

[15] RK. Durga, V. Gopika, RV. Sanyasi, et al.: Dynamic fault tree analysis using Monte Carlo simulation in probabilistic safety assessment. Reliab Eng Syst Safety 2009; 94(4):872-83.

[16] B. W. Johnson.: Design and Analysis of Fault Tolerant Digital Systems. Reading, MA: Addison-Wesley, 1989.

[17] H. Chen, J. Han.: Stochastic Computational Models for Accurate Reliability Evaluation of Logic Circuits. Proc. Great Lakes Symp. VLSI (GLVLSI), Providence, RI, USA, pp. 61-66 (2010).

[18] J. Han, H. Chen, J. Liang, P. Zhu, Z. Yang and F. Lombardi.: A Stochastic Computational Approach for Accurate and Efficient Reliability Evaluation. IEEE Transactions on Computers, vol. 63, no. 6, pp. 1336 - 1350, 2014.

[19] H. Aliee and H. R. Zarandi.: A Fast and Accurate Fault Tree Analysis Based on Stochastic Logic Implemented on FieldProgrammable Gate Arrays. IEEE Trans on reliability volume: 62, issue: 1, page(s): 13 - 22, March 2013.

[20] P. Zhu, J. Han, L. Liu and M. J. Zuo.: A Stochastic Approach for the Analysis of Fault Trees with Priority AND Gates. IEEE Transactions on Reliability, vol. 63, no. 2, pp. 480 - 494, June 2014.

[21] A. Ejlali, and S. Miremadi (2004).: FPGA-based Monte Carlo simulation for fault tree analysis. Microelectronics Reliability 44(6), 1017-1028

[22] G. Merle.: Improving the Efficiency of Dynamic Fault Tree Analysis by Considering Gates FDEP as Static. European Safety and Reliability conference 2010.

[23] S. J. Bavuso.: A novel solution-technique applied to a novel WAAS architecture. In Proceedings of Annual Reliability and Maintainability Symposium (RAMS'98), USA, Jan. 1998, pp. 229-234.

[24] L. Xing, A. Shrestha, L. Meshkat, and W. Wang.: Incorporating Common-Cause Failures into the Modular Hierarchical Systems Analysis. IEEE TRANSACTIONS ON RELIABILITY, VOL. 58, NO. 1, MARCH 2009.

[25] L. Xing and W. Wang.: Probabilistic common-cause failures analysis, in Proc. of the 54th Annual Reliability \& Maintainability Symposium ,Las Vegas, NV, USA, Jan. 2008.

[26] M. Stamatelatos, and W. Vesely (2002).: Fault tree handbook with aerospace applications. Volume 1.1, pp. 1-205. NASA Office of Safety and Mission Assurance.

[27] J. D. Andrews and J. B. Dugan.: Dependency modeling using fault tree analysis. In proceedings of the 17 th International 
System Safety Conference, USA, Aug. 1999.

[28] J. B. Dugan and S. A. Doyle.: New Results in Fault Tree Analysis, Tutorial notes of Annual Reliability \& Maintainability Symposium, (Jan.) 1997.

[29] K. B. Misra (Editor).: Handbook of Performability Engineering, Springer-Verlag, London, ISBN: 978-1-84800-130-5, (Oct.) 2008.

[30] J. B. Dugan, S. J. Bavuso, and M. A. Boyd.: Dynamic fault-tree models for fault-tolerant computer systems. IEEE Trans. Rel., vol. 41, no. 3, pp. 363-377, Sep. 1992.

[31] J. She and M. Pecht.: Reliability of a k-out-of-n Warm-Standby System. IEEE Trans. Reliability, vol. 41, no. 1, 1992.

[32] B. R. Gaines.: Stochastic Computing Systems. Advances in Information Systems Science, Vol. 2, pp. 37-172, 1969.

[33] Y. S. Dai, M. Xie, K. L. Poh, and S. H. Ng.: A model for correlated failures in N-version programming, IIE Trans., vol. 36, no. 12, pp.1183-1192, 2004.

[34] K. N. Fleming and A. Mosleh.: Common-cause data analysis and implications in system modeling, in Proc. of the Intl. Topical Meeting on Probabilistic Safety Methods and Applications, Feb. 1985, vol. 1, pp.3/1-3/12, EPRI NP-3912-SR.

[35] Z. Tang, H. Xu, and J. B. Dugan.: Reliability analysis of phased mission systems with common cause failures, in Proc. of the 51st Annual Reliability and Maintainability Symposium, Jan. 2005, pp. 313-318.

[36] J. K. Vaurio.: An implicit method for incorporating common-cause failures in system analysis, IEEE Trans. Reliability, vol. 47, no. 2, pp.173-180, June 1998.

[37] L. Xing, L. Meshkat, and S. Donohue.: An efficient approach for the reliability analysis of phased-mission systems with dependent failures, in Proc. of the 8th Intl. Conf. on Probabilistic Safety Assessment and Management (PSAM8), New Orleans, LA, USA, May 14-19, 2006.

[38] L. B. Page and J. E. Perry.: A model for system reliability with common-cause failures, IEEE Trans. Reliability, vol. 38, no. 4, pp. 406-410, Oct. 1989.

[39] C. P. Robert and G. Casella, Monte Carlo Statistical Methods. Springer, 2004.

[40] J. Han, E. Boykin, H. Chen, J. Liang and J. Fortes.: On the Reliability of Computational Structures using Majority Logic. IEEE Transactions on Nanotechnology, vol. 10, no. 5, pp. 1099-1112, September 2011.

[41] G. Merle, J. M. Roussel, J. J. Lesage.: Dynamic fault tree analysis based on the structure function. In Annual reliability and maintainability symposium 2011, Lake Buena Vista, 2011, p.462-467. 


\section{Author biographies:}

Peican Zhu received the B.S. degree in 2008 and the M.Sc. degree in 2011, both from the Northwestern Polytechnical University (NWPU), Xi'an, Shaanxi, China. He is currently working towards the Ph.D. degree in the Department of Electrical and Computer Engineering, University of Alberta, Edmonton, AB, Canada.

His current research interests include stochastic computational models for system reliability analysis, gene network models and pathway analysis. He is a recipient of the Alberta Innovates Graduate Student Scholarship.

Jie Han received the B.Sc. degree in electronic engineering from Tsinghua University, Beijing, China, in 1999 and the Ph.D. degree from Delft University of Technology, The Netherlands, in 2004. He is currently an assistant professor in the Department of Electrical and Computer Engineering at the University of Alberta, Edmonton, AB, Canada.

His research interests include reliability and fault tolerance, nanoelectronic circuits and systems, novel computational models for nanoscale and biological applications. Dr. Han was nominated for the 2006 Christiaan Huygens Prize of Science by the Royal Dutch Academy of Science (Koninklijke Nederlandse Akademie van Wetenschappen (KNAW) Christiaan Huygens Wetenschapsprijs). His work was recognized by the 125 th anniversary issue of Science, for developing theory of fault-tolerant nanocircuits. Dr. Han served as a General Chair and Technical Program Chair in IEEE International Symposium on Defect and Fault Tolerance in VLSI and Nanotechnology Systems (DFTS) 2013 and 2012, respectively, and as a Technical Program Committee Member in several other international symposia and conferences.

Leibo Liu received the B.S. degree in electronic engineering from Tsinghua University, Beijing, China, in 1999 and the Ph.D. degree in Institute of Microelectronics, Tsinghua University, in 2004. He currently serves as an Associate Professor in Institute of Microelectronics, Tsinghua University. His research interests include Reconfigurable Computing, Mobile Computing and VLSI DSP. Dr. Liu has published more than 70 refereed papers, and served as TPC member or reviewers for several international key conferences and leading journals.

Fabrizio Lombardi (M'81-SM'02-F'09) graduated in 1977 from the University of Essex (UK) with a B.Sc. (Hons.) in Electronic Engineering. In 1977 he joined the Microwave Research Unit at University College London, where he received the Master in Microwaves and Modern Optics (1978), the Diploma in Microwave Engineering (1978) and the Ph.D. from the University of London (1982).

He is currently the holder of the International Test Conference (ITC) Endowed Chair Professorship at Northeastern University, Boston. His research interests are bio-inspired and nano manufacturing/computing, VLSI design, testing, and fault/defect tolerance of digital systems. He has extensively published in these areas and coauthored/edited seven books. 